\title{
MiR-92a regulates endothelial progenitor cells (EPCs) by targeting GDF11 via activate SMAD2/3/FAK/Akt/eNOS pathway
}

\author{
Hai-Tao Huang ${ }^{1 \#}$, Zhen-Chuan Liu ${ }^{2 \#}$, Kai-Qin $\mathrm{Wu}^{2}$, Shao-Rui Gu ${ }^{2}$, Tian-Cheng Lu ${ }^{2}$, Chong-Jun Zhong ${ }^{1}$, \\ Yong-Xin Zhou ${ }^{2}$ \\ ${ }^{1}$ Department of Thoracic and Cardiovascular Surgery, Nantong First People's Hospital, Nantong 226001, China; ${ }^{2}$ Department of Thoracic and \\ Cardiovascular Surgery, Tongji Hospital, School of Medicine, Tongji University, Shanghai 200065, China \\ Contributions: (I) Conception and design: HT Huang, ZC Liu, YX Zhou; (II) Administrative support: YX Zhou; (III) Provision of study materials or \\ patients: HT Huang, ZC Liu, KQ Wu; (IV) Collection and assembly of data: SR Gu, TC Lu, CJ Zhong; (V) Data analysis and interpretation: HT \\ Huang, ZC Liu; (VI) Manuscript writing: All authors; (VII) Final approval of manuscript: All authors. \\ \#These authors contributed equally to this work. \\ Correspondence to: Yong-Xin Zhou. Department of Thoracic and Cardiovascular Surgery, Tongji Hospital, School of Medicine, Tongji University, No. \\ 389 Xincun Road, Shanghai 200065, China. Email: zhou6302@tongji.edu.cn.
}

Background: The effects of miR-92a on EPCs are still poorly elucidated. This study aimed to investigate the effects of miR-92a on EPCs (Endothelial progenitor cells) in a model of hypoxia (HO) or high glucose (HG)-induced EPCs injury by targeting GDF11 (Differentiation growth factor 11).

Methods: The effects of miR-92a on EPCs subjected to HO or HG were investigated firstly. Subsequently, the action mechanism of miR-92a on EPCs by targeting GDF11 was elucidated. Proliferation, apoptosis, migration, angiogenesis was measured with MTT, flow cytometry, transwell, tube formation respectively. After $24 \mathrm{~h}$, levels of reactive oxygen species (ROS) were measured by fluorescence intensity. LDH and NO (nitric oxide) levels were determined by ELISA. The expression of FLK-1 (fetal liver kinase 1) and vWF (von Willebrand factor) was detected by immunofluorescence. mRNA and protein expression levels were examined using PCR and western blotting respectively. The interaction between miR-92a and GDF11 was evaluated by dual-luciferase reporter assay.

Results: Our results showed that HO or HG increased apoptosis, production of LDH and generation of ROS, but decreased the ability of migration and tube formation and generation of NO in EPCs; inhibiting of miR-92a decreased HO or HG-induced injury of EPCs, whereas miR-92a over-expression had the opposite effect; the protective effects induced by inhibiting of miR-92a on EPCs could be reversed by GDF11 siRNA and the harmful effects induced by over-expression of miR-92a could be rescued by over-expression of GDF11, which showed that the harmful effects of miR-92a be related to its inhibition of GDF11 and subsequent inactivation of the SMAD2/3/FAK/Akt/eNOS signaling pathway.

Conclusions: Inhibiting miR-92a can protect EPCs from HO or HG-induced injury. The effect of miR92a on EPCs are mediated by regulating of GDF11 and downstream SMAD2/3/FAK/Akt/eNOS signaling pathway.

Keywords: Endothelial progenitor cells (EPCs); miR-92a; differentiation growth factor 11 (GDF11); reactive oxygen species (ROS); nitric oxide (NO)

Submitted May 08, 2019. Accepted for publication Aug 26, 2019.

doi: 10.21037/atm.2019.09.35

View this article at: http://dx.doi.org/10.21037/atm.2019.09.35 


\section{Introduction}

Endothelial progenitor cells (EPCs) play an important role in promoting angiogenesis, re-endothelialization and repairing damaged vessels (1). Asahara et al. reported that EPCs mobilized from bone marrow were involved in regeneration of neovascularization and repair of vascular endothelial cells in myocardial infarction site (2).

Most patients with coronary artery disease (CAD) are complicated with diabetes mellitus (DM), hyperlipidemia, hypertension and so on. A number of studies have indicated that risk factors for CAD such as advanced age, high blood glucose and hyperlipidemia inhibit the mobilization and impair the function of circulating EPCs (3-6).

GDF11 a member of transforming growth factor $\beta$ superfamily, play a role mainly by canonial SMAD2/3 signal pathway (7). Clinical studies indicated that the level of GDF11 in peripheral blood was closely related to cardiovascular events and related mortality $(8,9)$. Finkenzeller et al. also reported that GDF11 could induce migration and tube formation of EPCs isolated from human peripheral blood in vitro (10). Recently, animal experiment further verified that GDF11 could improve the function of EPCs in diabetic animals and promote vascular regeneration and repair (11).

MicroRNAs (miRNAs) are group of short noncoding RNA molecules (approximately composed of 22 nucleotides) and regulate quit a lot of pathophysiological processes by binding the 3'-untranslated regions of mRNA (12). miR-92a, a member of miR-17 92a cluster, exerts anti-angiogenesis function by targeting a series of genes such as integrin subunits $\alpha 5$ (ITGA5) and $\alpha \mathrm{v}$ (ITGAV), While inhibition of miR-92a improved angiogenesis in models of hind limb- or myocardial ischaemia (13). Recently, miR-92a has been extensively researched in several cancers. Ou et al. found that miR-92a up-regulated by estrogen receptor $\beta$ (ER $\beta$ ) could decrease the expression of DOC-2/DAB-2 interacting protein (DAB2IP) a tumor suppressor in human bladder cancer (12). Casadei et al. demonstrate that miR-92a-3p could be secreted by liposarcoma cells by extracellular vesicles and stimulated secretion of proinflammatory cytokine IL-6 which in turn promoted the proliferation, invasion, and metastasis of liposarcoma cell (14).

The expression of miR - 92a was increased in ECs with or without diabetic (15). Over-expression of miR-92a impaired the endothelial function and suppressed the expression of HO-1 in ECs. The oxidative stress was inhibited and the endothelial function was improved by down-expression of miR-92a through promoting the expression and activity of HO-1 in aortas of diabetic mouse (16).

It was verified that miR-92a expressed in EPCs, and the expression increased in EPCs from CAD patients compared with healthy individuals (17). However, the effects of miR92a on EPCs are still poorly elucidated. Previous studies indicated that GDF11 can improve the function of EPCs under these pathological conditions and it's predicted that miR-92a has targeting interaction with GDF11. Consequently, we hypothesized that inhibiting miR-92a can exert protective action on EPCs by up-regulation of GDF11. In this study, the effects of miR-92a on EPCs under various circumstance and the relationship between miR-92a and GDF11 in EPCs were investigated.

\section{Methods}

\section{Cell culture and groups}

The whole study was approved by the Ethics Committee of Nantong First People's Hospital. EPCs isolated from bone marrow of Sprague-Dawley rats were maintained in M199 basic medium containing 10\% fetal bovine serum, $1 \%$ streptomycin and penicillin at $37^{\circ} \mathrm{C}$ with $5 \% \mathrm{CO}_{2}$. Cultured cells were identified by CD133 and VEGFR-2 immunofluorescence stain (picture not shown).

The effects of miR-92a on EPCs subjected to HO or HG were investigated firstly. EPCs were divided into 10 groups: EPCs (blank control); EPCs + NC (negative control); EPCs + miR-92a mimic; EPCs + miR-92a inhibitor; EPCs + HO + NC; $\mathrm{EPCs}+\mathrm{HO}+$ miR-92a mimic; $\mathrm{EPCs}+\mathrm{HO}+$ miR92a inhibitor; EPCs + HG + NC; EPCs + HG + miR-92a mimic; EPCs + HG + miR-92a inhibitor. Subsequently, the action mechanism of miR-92a on EPCs by targeting GDF11 was elucidated. EPCs were divided into 6 groups: EPCs (blank control); EPCs + miR-92a inhibitor; EPCs + miR-92a inhibitor + siNC (siRNA negative control); EPCs + miR-92a inhibitor + siGDF11 (siRNA GDF11); EPCs + miR-92a mimic; EPCs + miR-92a mimic + rGDF11 (recombinant GDF11).

EPCs in all groups were observed and counted in under light microscopy at least three different fields by two observers blind to the research group, and representative pictures were captured. The mean and standard deviation 
were calculated and analyzed $(\mathrm{n}=3)$.

\section{miRNA transfection and GDF11 silencing}

EPCs were digested with $0.25 \%$ trypsin when overgrew the bottom of flask. A single-cell suspension of $5 \times 10^{5}$ cells $/ \mathrm{mL}$ was seeded into 6 -well plates $(3 \mathrm{~mL} /$ well), and incubated at $37{ }^{\circ} \mathrm{C}$ with $5 \% \mathrm{CO}_{2}$ or with $1 \% \mathrm{O}_{2}$ (hypoxia), or with glucose (40 mM, high glucose). EPCs were transfected when grown to $80 \%$ confluence.

Cells were divided into blank control, NC, miR-92a mimic and miR-92a inhibitor groups under the condition of HO or HG. In the blank control group, cells were maintained in serum-free M199 medium without other treatment. miR-92a mimics or inhibitor or NC (Life Science) were transfected in the presence of Lipofectamine ${ }^{\circledR}$ 2000 (Thermo Fisher Scientific, Carlsbad, CA, USA) at $37^{\circ} \mathrm{C}$ for $4-6 \mathrm{~h}$ following the manufacturer's protocol. MiR-92a inhibitor was applied to knockdown the expression of miR-92a and the mimic was to over-express miR-92a in EPCs. RT-PCR was used to evaluate the transfection efficiency.

To investigate the role of GDF11 in regulatory function of miR-92a in EPCs, GDF11 expression was knockdown in EPCs by GDF11 siRNA or over-expressed by rGDF11. Western blotting and RT-PCR were performed to confirm the knockdown or over-expression efficiency.

\section{Real-time PCR (RT-PCR)}

To investigate the efficiency of knockdown and overexpression by inhibitor or siRNA and mimic or recombinant respectively, RT-PCR was used to detect the mRNA expression of miR-92a and GDF11. The expression of surface adhesion molecules were also detected by RT-PCR. Total RNA was extracted from EPCs with TRIzol ${ }^{\circledR}$ reagent (Invitrogen, Waltham, MA, USA). cDNA was generated from total RNA using a cDNA reverse transcription kit (Thermo, \#K1622, USA) with specific primers for miR-92a [5' ACACTCCAGCTGGGAGGTTGGGATTTGTCGC 3' (forward), 5' CTCAACTGGTGTCGTGGAGTC GGCAATTCAGTTGAGA 3' (reverse)], small nuclear RNA U6 [5' CTCGCTTCGGCAGCACA 3' (forward), 5' AACGCTTCACGAATTTGCGT 3' (reverse)] and gdf11 [5' CAGCCCTCTGTGTTGCCATTTCCA 3' (forward), 5' CACCCCCTCCCCCTGCGGTCCCTT 3' (reverse)] PECAM-1 [5' CACAGACAAGCCCACCAGAGACAT
3' (forward), 5' TTCACAGAGCACCGAAGCACCATT 3' (reverse)] ICAM-1 [5' CGGTGCTCAGGTATCCATCCATCCC 3' (forward), 5' AGTTCGTCTTTCATCCAGTTAGTCT 3' (reverse)] VCAM-1 [5' GAAAAAGAAAGCCAAGACAGGAGA 3' (forward), 5' GCAAGGAGTTCAGGGGAAAAATAG 3' (reverse)] Gapdh [5' TGCTGGTGCTGAGTATGTCGTGGAG 3' (forward), 5' GGGGCGGAGATGATGACCCTTTTGG 3' (reverse)] (Shanghai Sangon Biotech Co., Ltd., Shanghai, China).

Quantitative real-time PCR was performed with SYBR green assay kit (Thermo Fisher) and Reverse transcription kit (Thermo Fisher). The primer sequences listed above were designed according to the cDNA sequences in GenBank. In total $20 \mu \mathrm{L}$ RT-PCR reaction mixture, it contained cDNA $1.0 \mu \mathrm{L}, 10 \mathrm{mM}$ each paired primer $1.0 \mu \mathrm{L}$, and SybrGreen qPCR Master Mix $(2 \times) 10 \mu \mathrm{L}$, $\mathrm{ddH}_{2} \mathrm{O} 7.0 \mu \mathrm{L}$. The amplification temperature was set at $94^{\circ} \mathrm{C}$ and amplification cycle was set at 40 cycles. Relative mRNA levels were normalized to those of the housekeeping gene GAPDH and analyzed with the comparative threshold cycle $(\mathrm{Ct})$ method with arithmetic formula $2^{\wedge}$ (-Delta Delta CT). Statistical analysis was performed using the MannWhitney $\mathrm{U}$ test at a $95 \%$ confidence interval.

\section{MTT assay}

Cell proliferative activity was detected by MTT (Sigma Aldrich) assay. EPCs were seeded in 96-well plates at a density of $5 \times 10^{4}$ cells $/ \mathrm{mL}$ (100 $\mu \mathrm{L} /$ well). Ten $\mu \mathrm{L}$ of MTT solution was added to each well on the 1 st to 5 th days after treatment. Followed by $4 \mathrm{~h}$ incubation at $37^{\circ} \mathrm{C}$, EPCs were lysed with $200 \mu \mathrm{L}$ DMSO and shook for $10 \mathrm{~min}$. The OD (optical density) value at $492 \mathrm{~nm}$ was detected in enzymelabeled instrument (Thermo MK3 Type). All samples were tested in triplicates and the differences among groups were analyzed with statistical tools.

\section{Migration test}

The migration assay was performed in a 24-well transwell plate fitted with multi-porous polycarbonate membrane (8- $\mu \mathrm{m}$ pore size. Costar, Coring Incorporated, USA). Briefly, after transfection for $24 \mathrm{~h}$, EPCs were resuspended in serum-free media at a density of $1 \times 10^{5}$ cells $/ \mathrm{mL}$. $300 \mu \mathrm{L}$ of cell suspension was added to the top chamber, and the lower chamber was filled with $0.6 \mathrm{~mL}$ of M199 supplemented with $10 \% \mathrm{FBS}$. After incubation at $37^{\circ} \mathrm{C}$ with 
$5 \% \mathrm{CO}_{2}$ or hypoxia condition for $72 \mathrm{~h}$, nonmigrating EPCs on the upper side of the filter were removed with cotton swabs, the chambers were rinsed two times with PBS, and fixed with $4 \%$ paraformaldehyde for $30 \mathrm{~min}$, and stained with $0.1 \%$ crystal violet for $10 \mathrm{~min}$. Migrated cells were counted under microscope in triplicates, and the differences were performed statistical analysis.

\section{Angiogenesis assay (tube formation)}

The in vitro angiogenic potential of EPCs was performed by matrigel tube formation assay. Briefly, 24-well plates were coated with matrigel $(250 \mu \mathrm{L} /$ per well, BD Biosciences, USA). After transduced, EPCs were digested and adjusted to a concentration of $2 \times 10^{5}$ cell/ $/ \mathrm{mL}$, consequently $5 \times 10^{4}$ cell/well was seed, following incubating at $37{ }^{\circ} \mathrm{C}$ with $5 \% \mathrm{CO}_{2}$ or hypoxia for $6 \mathrm{~h}$ to form tubes. Capillary-like structures were imaged and counted.

\section{Flow cytometry of apoptotic cells}

After transfection for $24 \mathrm{~h}$ in various groups, apoptotic cells were assessed by with the Annexin V-FITC/propidium iodide (PI) apoptosis detection kit (Beyotime Biotech, Jiangsu, China) according to the manufacturer's instructions. Briefly, the cells were rinsed with PBS $\left(4^{\circ} \mathrm{C}\right)$ once and re-suspended with $300 \mu \mathrm{L}$ binding buffer following adding $5 \mu \mathrm{L}$ Annexin V-FITC and $10 \mu \mathrm{L}$ PI. The EPCs mixture was incubated for $10 \mathrm{~min}$ at room temperature shielded from light. Flow cytometry (BD-FACSVerse, USA) was performed to detect the cell apoptosis and CellQuest software (Becton Dickinson) were used to analyze the data.

\section{Immunofluorescence (IF) test}

After transfection for $24 \mathrm{~h}$ in various groups and rinsed with PBS once, the EPCs were fixed cells with $4 \%$ paraformaldehyde, penetrated with $0.5 \%$ TritionX-100\%, Blocked with $5 \%$ FBS for $1 \mathrm{~h}$ at room temperature. EPCs were incubated with FLK-1 (1:500; 26415-1-AP, Proteintech, USA), vWF (1:500; 11778-1AP, Proteintech, USA) at $4{ }^{\circ} \mathrm{C}$ overnight. Consequently, the EPCs incubated with the second antibody Goat Anti-Rabbit IgG (Beyotime Institute of Biotechnology, China) (1:200 dilution) for 1 hour at $37^{\circ} \mathrm{C}$. The nuclei was counterstained with Hoechst (Beyotime) for $15 \mathrm{~min}$ at room temperature shielded from light. The EPCs were observed and photographed by fluorescent microscopy.

\section{ROS measurements by ROS fuorescent probe-DHE}

The intracellular ROS level was detected by ROS Fluorescent Probe-DHE (Dihydroethidium) (Beyotime Biotech). Briefly, EPCs were incubated with DHE $(10 \mu \mathrm{mol} / \mathrm{L})$ at $37^{\circ} \mathrm{C}$ for $20 \mathrm{~min}$ and rinsed three times by serum-free cell culture medium to wipe off redundant DHE. DHE fluorescence intensity was detected by inverted fluorescence microscope and the representative photographs were taken (Olympus, IX71, Japan).

\section{Detection of LDH and NO by ELISA (enzyme-linked immunosorbent assay)}

Supernatant NO and $\mathrm{LDH}$ concentration in various groups were detected by NO assay kit (Microwell plate method) and LDH assay kit (Microwell plate method) (Nanjing Jiancheng Bioengineering Institute, Nanjing, China) respectively, following the manufacturer's protocols. Optical density (OD) was then measured in enzyme-labeled instrument (Thermo MK3 Type) at a wavelength of $550 \mathrm{~nm}$ for $\mathrm{NO}$ and $450 \mathrm{~nm}$ for LDH. The results were calculated according to the manufacturer's formula.

\section{Western blotting}

EPCs were lysed in RIPA buffer with PMSF (Beyotime). Proteins were extracted following the manufacturer's protocol (Beyotime Biotech). After protein quantification by BCA assay kit (Beyotime Biotech), proteins were separated using $10 \%$ sodium dodecyl sulfate polyacrylamide gel electrophoresis gels (SDS-PAGE) and subsequently transferred onto polyvinylidene fluoride (PVDF) membranes. After blocking, the membranes were incubated with primary antibody against GDF11 $(1 \mu \mathrm{g} / \mathrm{mL}$; MAB788100, R\&D, USA), eNOS (1:500; Ab76198, Abcam, Cambridge, MA, USA), p-eNOS (1:500; Ab138430, Abcam), p-SMAD2/3 (1:500; ab63399, Abcam), SMAD2/3 [1:1000; CST8685S, Cell Signaling Technology (CST), Danvers, MA, USA], p-Akt (1:2000; CST4060S, CST), AKT(1:1000; 10176-2-AP, Proteintech, USA), GAPDH (1:5000; 60004-I-IG, Proteintech, USA) overnight at $4{ }^{\circ} \mathrm{C}$, followed by incubation with goat anti-rabbit, goat anti- 
mouse HRP-conjugated secondary antibody (1:5000; Proteintech, USA) for $2 \mathrm{~h}$ at room temperature. Staining intensity of the bands was visualized using ECL reagents (ECL-0011, Beijing, Dingguo, China), and detected by the Integrated chemiluminescence apparatus (ChemiScope 5300 Pro, Clinx, Shanghai, China).

\section{Dual-luciferase reporter assay}

The interaction between GDF11 and miR-92a was investigated by dual-luciferase reporter assay system (Promega E1910, Madison, WI, USA). The rat GDF11 sequence was obtained from PUBMED (Gene ID: 29454), and analysis indicated an miR-92a binding site in the 3' untranslated region (UTR) of GDF11 sequence (CCCCACC). Then, the 3' UTR of GDF11 sequence was introduced to the pmirGLO luciferase vector (Promega, Madison, WI, USA). pmirGLO-GDF11-WT (wild type: CCCCACC) and pmirGLO-GDF11-MUT (mutation: GGGGTGG) were constructed. Next, the single-cell suspension $\left(5 \times 10^{4}\right.$ cells $\left./ \mathrm{mL}\right)$ was added to 96 -well plates, and incubated at $37^{\circ} \mathrm{C}$ for $24 \mathrm{~h}$. EPCs were transfected with miR-92a mimic and miR-92a inhibitor respectively in the presence of Lipofectamine ${ }^{\circledR} 2000$. EPCs were then treated with pmirGLO-GDF11-WT or MUT vector $(1.5 \mu \mathrm{g}$ in $250 \mu \mathrm{L}$ serum-free culture medium) or blank vector in each group respectively. After incubation for $6 \mathrm{~h}$, the supernatant was refreshed with $100 \mu \mathrm{L}$ complete medium, and incubated for another $48 \mathrm{~h}$. EPCs were rinsed with phosphate-buffered saline (PBS) twice. EPCs were incubated on shaking table at room temperature for $15 \mathrm{~min}$, after adding $100 \mu \mathrm{L}$ passive lysis buffer $(1 \times$ PLB, Promega). Then, $20 \mu \mathrm{L}$ lysed cell sample and $100 \mu \mathrm{L}$ LARII were added to each well of a 96-well plate, and RLU1 (relative light unit) was measured. Consequently, addition of $100 \mu \mathrm{L} 1 \times$ Stop\&Glo ${ }^{\circledR}$ Reagent, RLU2 (relative light unit) was measured. The ratio of RLU1 to RLU2 was calculated.

\section{Statistical analysis}

Statistical analysis was performed with SPSS version 20.0 for Windows. Data are expressed as the mean \pm standard deviation. Comparisons were performed with one-way analysis of variance among groups. The Mann-Whitney $U$ test at a $95 \%$ confidence interval was used to analyze the relative mRNA expression difference. A value of $\mathrm{P}<0.05$ was considered statistically significant.

\section{Results}

\section{The expression of miR-92a and GDF11 in EPCs under the condition of $\mathrm{HO}$ or $\mathrm{HG}$}

EPCs were successfully isolated from bone marrow of rats, and identified by the expression of CD133 and FLK-1 (VEGFR2) using IF test (data no show). Whether HO or HG affect the expression of miR-92a and GDF11 mRNA in EPCs, miR-92a and GDF11 mRNA expression in EPCs under various conditions was detected by RT-PCR (real time PCR). After the EPCs were subjected to $\mathrm{HO}$ or HG for $8 \mathrm{~h}$, miR-92a expression was increased (NC group in HO or HG $v s$. control). In HO and HG group, miR-92a expression was effectively improved by miR-92a mimic and inhibited by miR-92a inhibitor (Figure 1A). The level of GDF11 mRNA expression was reduced in EPCs which were then subjected to HO or HG (Figure 1B). The protein expression of GDF11 in EPCs under the condition of HO or HG was detected by Western blot. The protein expression of GDF11 was also decreased in EPCs subjected to $\mathrm{HO}$ or HG (Figure 1C). Results in this part indicated that $\mathrm{HO}$ or HG induced the expression of miR-92a and inhibited the expression of GDF11. HO or HG affect the expression of miR-92a and GDF11 in a contrary tendency.

\section{The effect of miR-92a on the proliferation, apoptosis, migration, and tube formation of EPCs under the condition of $\mathrm{HO}$ or $\mathrm{HG}$}

The proliferation of EPCs was detected by MTT assay. The proliferation of EPCs stimulated by hypoxia was inhibited in the following 5 days compared to untreated groups. No significant difference of proliferation was found in hypoxia groups except in $3^{\text {rd }}$ day after stimulated by miR-92a mimic or inhibitor. The proliferation of EPCs was improved by high glucose in the first 3 days, however, the proliferation effect was declining from $4^{\text {th }}$ day, when compared with untreated groups. miR-92a mimic or inhibitor had no effect on EPCs subjected to HG. These results indicated that over-expression or inhibition of miR-92a almost had no proliferation effect on EPCs subjected to HO or HG (Figure 2I).

The apoptosis of EPCs was detected by flow cytometry assay. The apoptosis rate of EPCs was increased significantly 
A

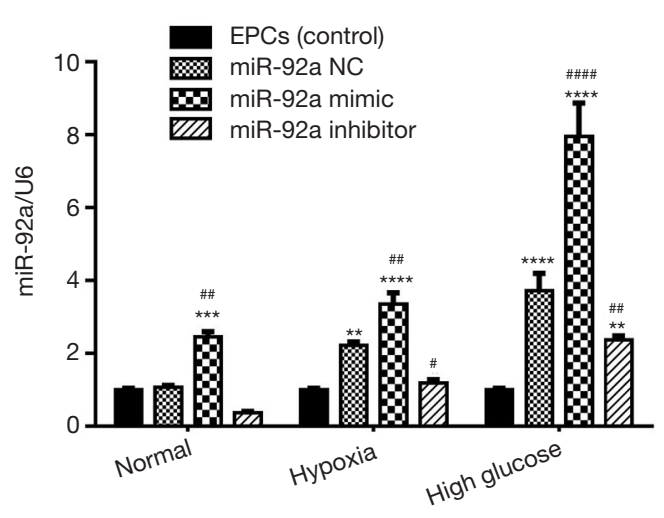

B

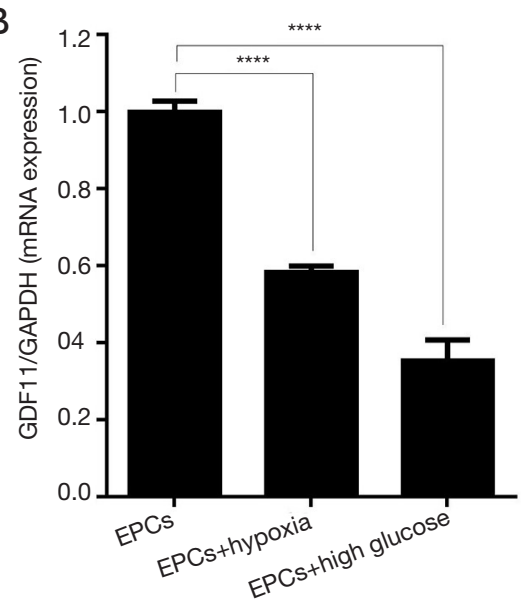

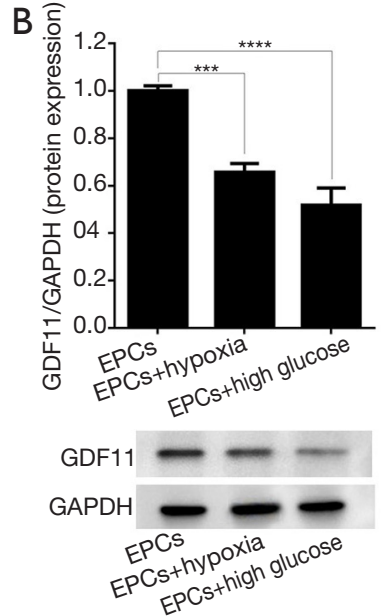

Figure 1 Efficiency of silencing and over-expression miR-92a expression after EPCs treated with miR-92a mimic or inhibitor and subjected to normal, HO or HG groups (A). GDF11 mRNA expression after EPCs subjected to untreated or HO or HG (B). GDF11 protein expression after EPCs subjected to untreated or HO or HG (C). ${ }^{* *}, \mathrm{P}<0.01,{ }^{* * *}, \mathrm{P}<0.001,{ }^{* * * *}, \mathrm{P}<0.0001$ vs. control group; ${ }^{*}, \mathrm{P}<0.05$, ${ }^{\# \#}$

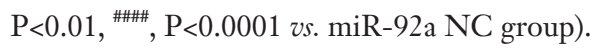

when treated by HO or HG. In untreated groups, miR-92a mimic promoted apoptosis when compared with control, $\mathrm{NC}$ or inhibitor groups. In HO treated groups, miR-92a inhibitor significantly ameliorated the apoptosis effect caused by hypoxia when compared with NC groups. In HG treated groups, miR-92a mimic significantly increased the apoptosis rate, however, miR-92a inhibitor inhibited the apoptosis rate compared with NC group. These results indicated that $\mathrm{HO}$ or $\mathrm{HG}$ can promote apoptosis of EPCs, and knockdown the expression of miR-92a can alleviate apoptosis of EPCs caused by HO or HG (Figure 2II).

The migration of EPCs was detected by transwell assay. The migration of EPCs of NC group in both HO and HG treatment was decreased compared with control group, which indicated that the migration of EPCs treated by HO or HG was inhibited significantly. In untreated groups, miR-92a inhibitor promoted migration, while miR-92a mimic inhibited migration compared with control or NC groups. Both in HO and HG treated groups, miR-92a inhibitor significantly improved the ability of migration compared with $\mathrm{NC}$ groups, miR-92a mimic aggravated the damage of migration. These results indicated that $\mathrm{HO}$ and HG can inhibit migration ability of EPCs, and inhibiting the expression of miR-92a can improve the migration ability of EPCs both in normal and pathological (HO and HG) condition (Figure 2III).

Angiogenesis ability of EPCs was detected by tube formation test. No significant difference was found between untreated and HO or HG groups. miR-92a mimic had no effect on EPCs in all groups. miR-92a inhibitor can improve angiogenesis ability of EPCs both in normal and pathological condition (Figure 2IV).

\section{The effect of miR-92a on the expression of adhesive molecules, LDH, NO, ROS, FLK-1, vWF of EPCs under the condition of $\mathrm{HO}$ or $\mathrm{HG}$}

The expression of pecam-1, icam-1, vcam-1 were detected by RT-PCR. The expression of pecam-1, icam-1, vcam-1 increased in EPCs subjected to HO or HG. Both miR92a mimic and inhibitor had no effect on the expression of pecam-1, icam-1, vcam-1 in EPCs under the condition of normal or pathology. These results indicated that HO and HG can promote the expression of pecam-1, icam-1, vcam-1, however, the effects can't be affected by knockdown or over-expression of miR-92a (Figure 3I A,B,C).

$\mathrm{LDH}$ and $\mathrm{NO}$ in EPCs culture supernatant were examined by ELISA. The level of LDH was elevated in EPCs culture supernatant subjected to HO or HG, which indicated that EPCs was impaired by HO or HG. miR-92a inhibitor inhibited the production of $\mathrm{LDH}$, while miR-92a mimic increase the LDH level in all three groups compared with miR-92a NC group (Figure 3I D). The concentration of $\mathrm{NO}$ was lower in EPCs culture supernatant subjected to HO or HG compared with untreated group. miR92a inhibitor could improve the production of $\mathrm{NO}$, while 


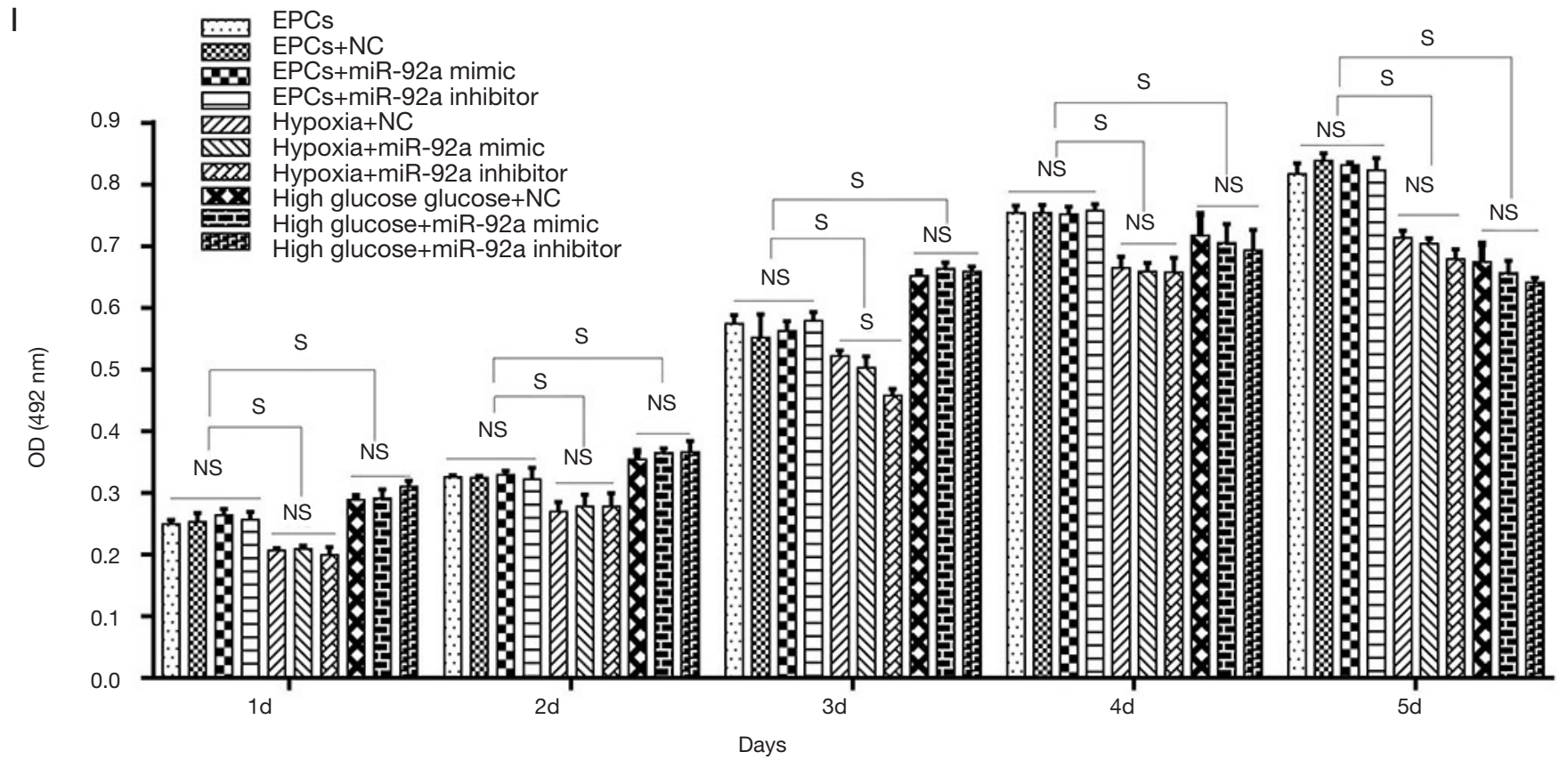

II
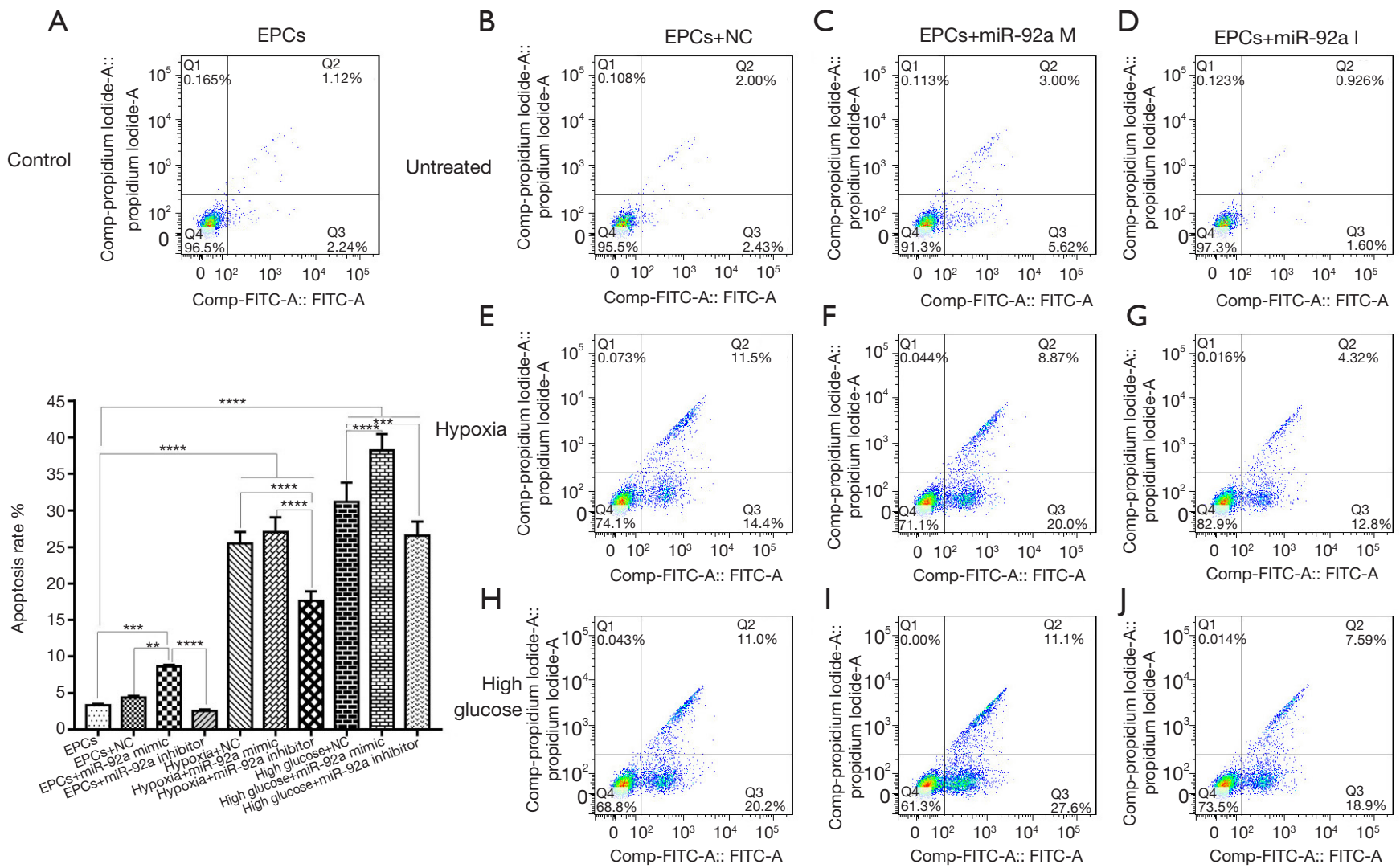

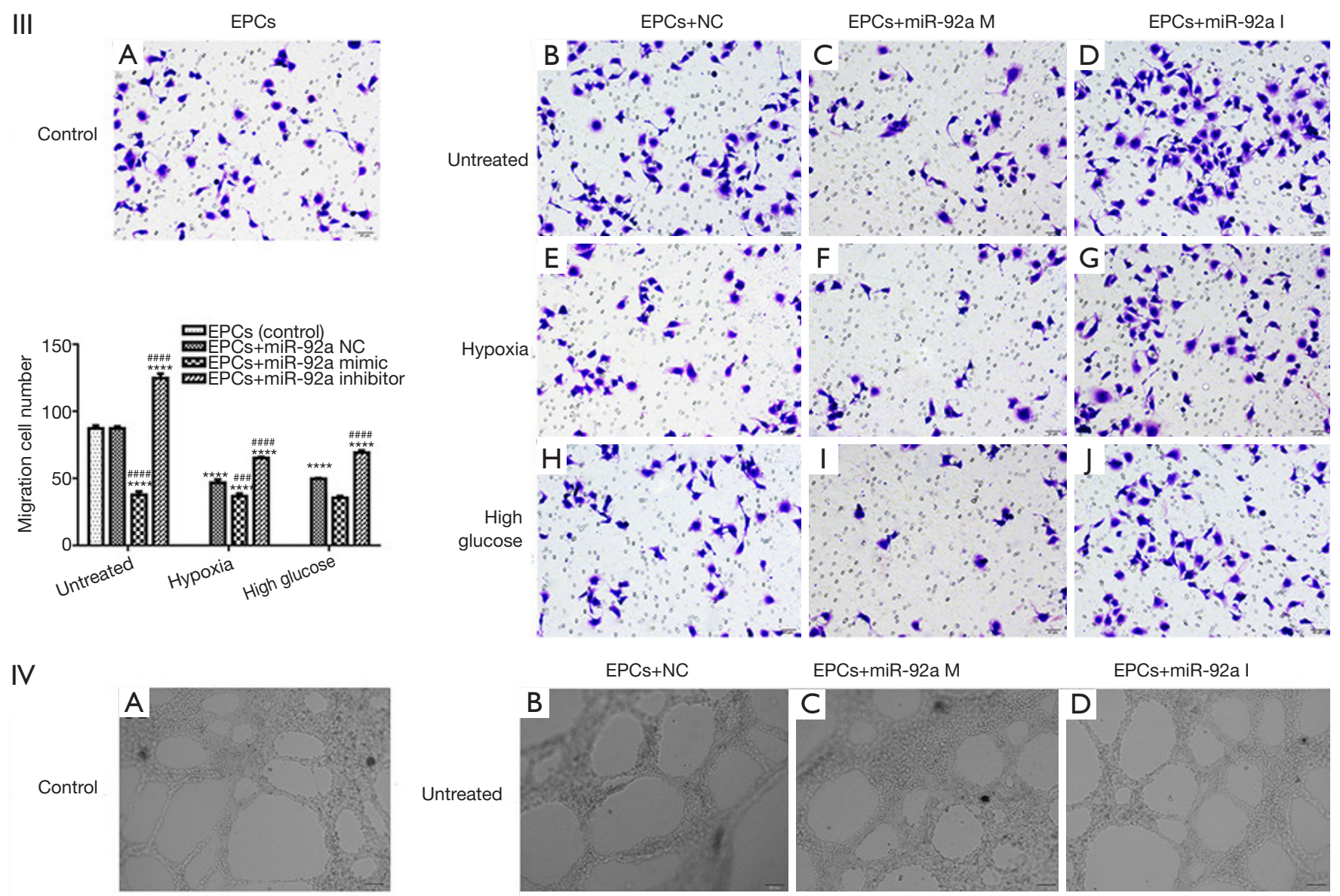

EPCs+miR-92a I
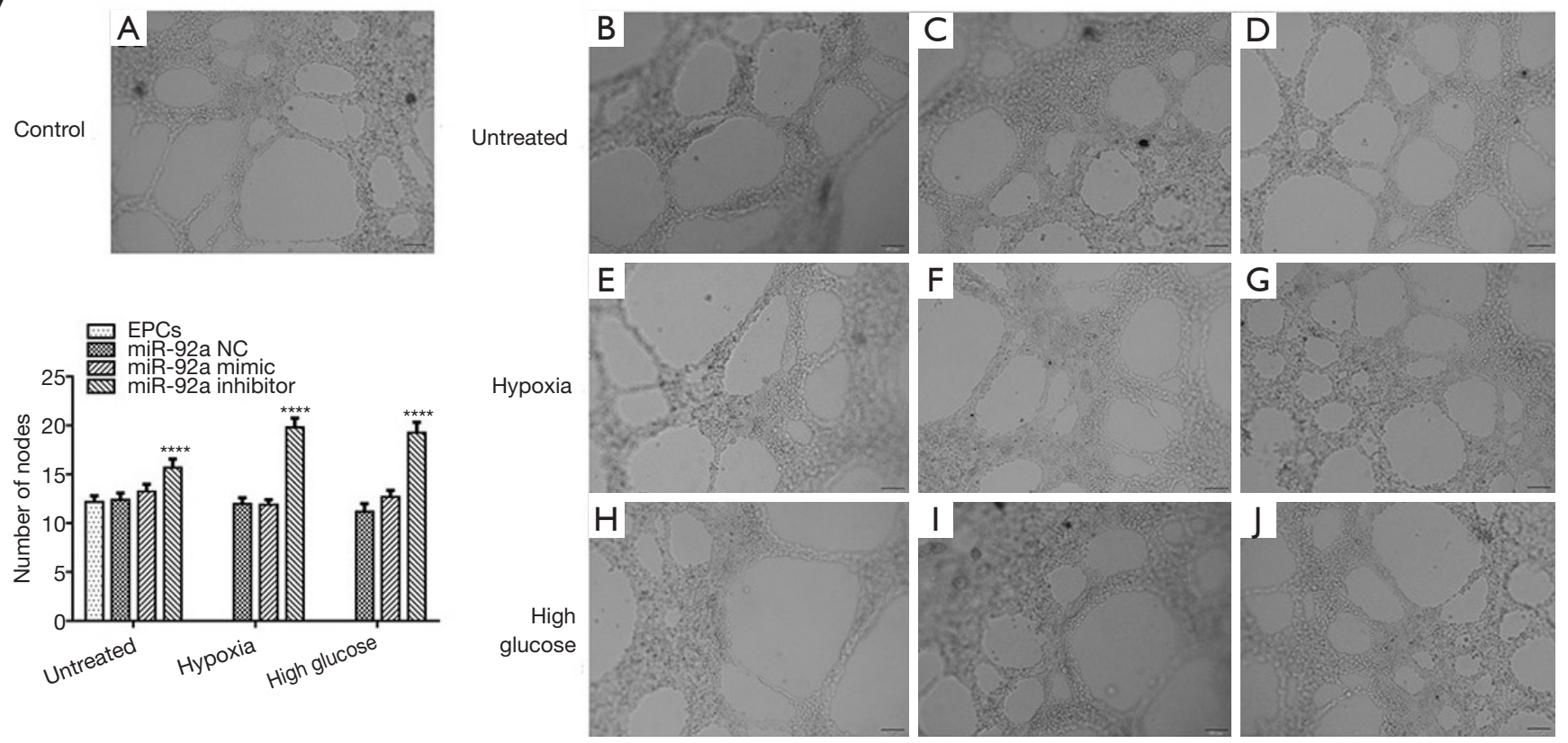

Figure 2 Cell proliferation, apoptosis, migration, tube formation after various treatments EPCs underwent miR-92a inhibition or miR-92a over-expression under the condition of HO or HG. I: MTT assay was applied to detected proliferation of EPCs (S: significant difference; NS: no significant difference). II: Cells were harvested for the detection of apoptotic cells by flow cytometry $\left.{ }^{* * *}, \mathrm{P}<0.001,{ }^{* * * *} \mathrm{P}<0.0001\right)$. III: migration ability of EPCs was tested by transwell assay (****, $\mathrm{P}<0.0001$ vs. control group; ${ }^{\# \# \#, ~} \mathrm{P}<0.001,{ }^{\# \# \#, ~} \mathrm{P}<0.0001$ vs. miR-92a $\mathrm{NC}$ group). (0.1\% crystal violet, $200 \times)$. IV: angiogenesis was evaluated by tube formation test (***, $\mathrm{P}<0.0001$ vs. control group and miR-92a $\mathrm{NC}$ group). 

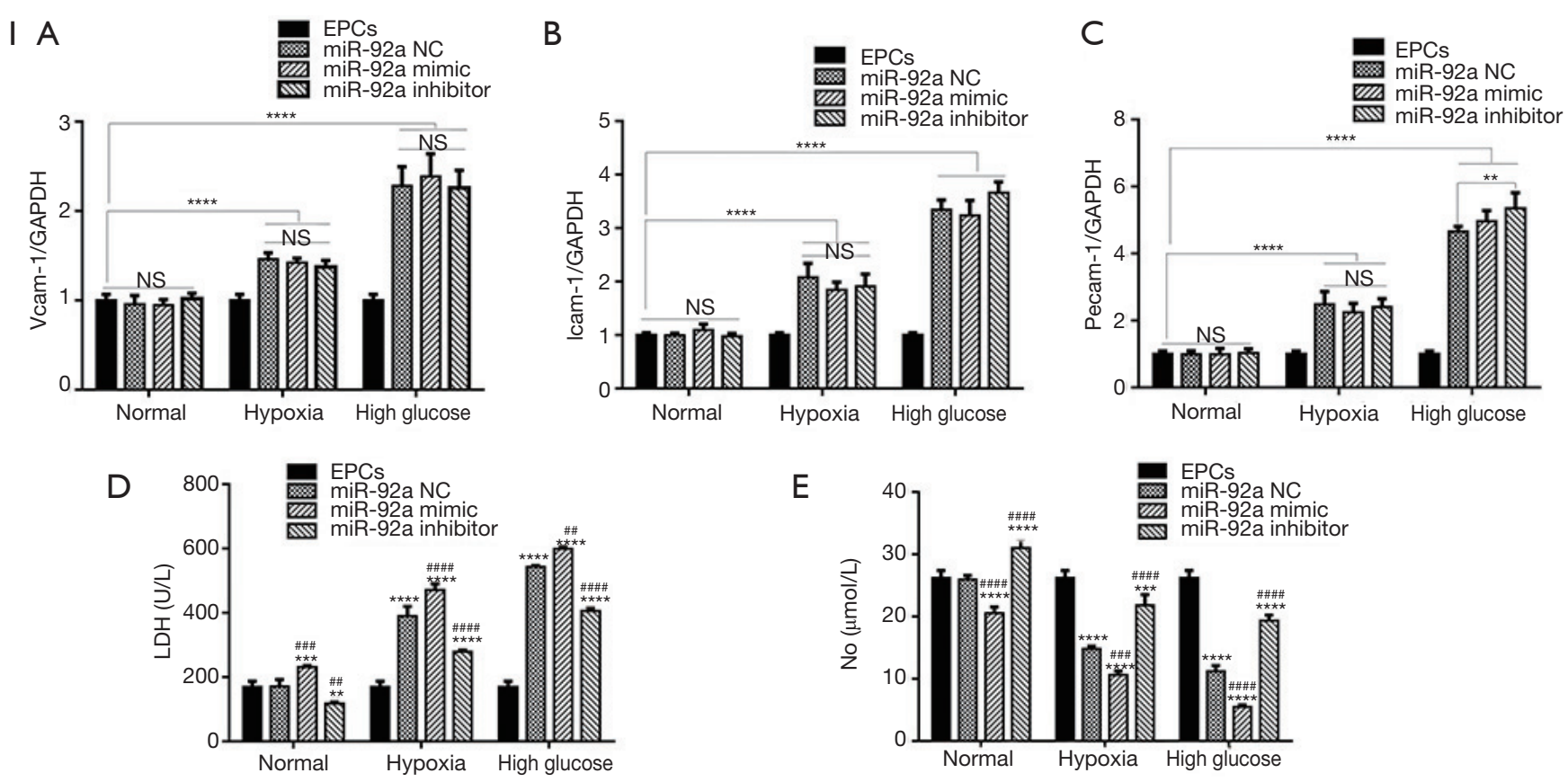

II
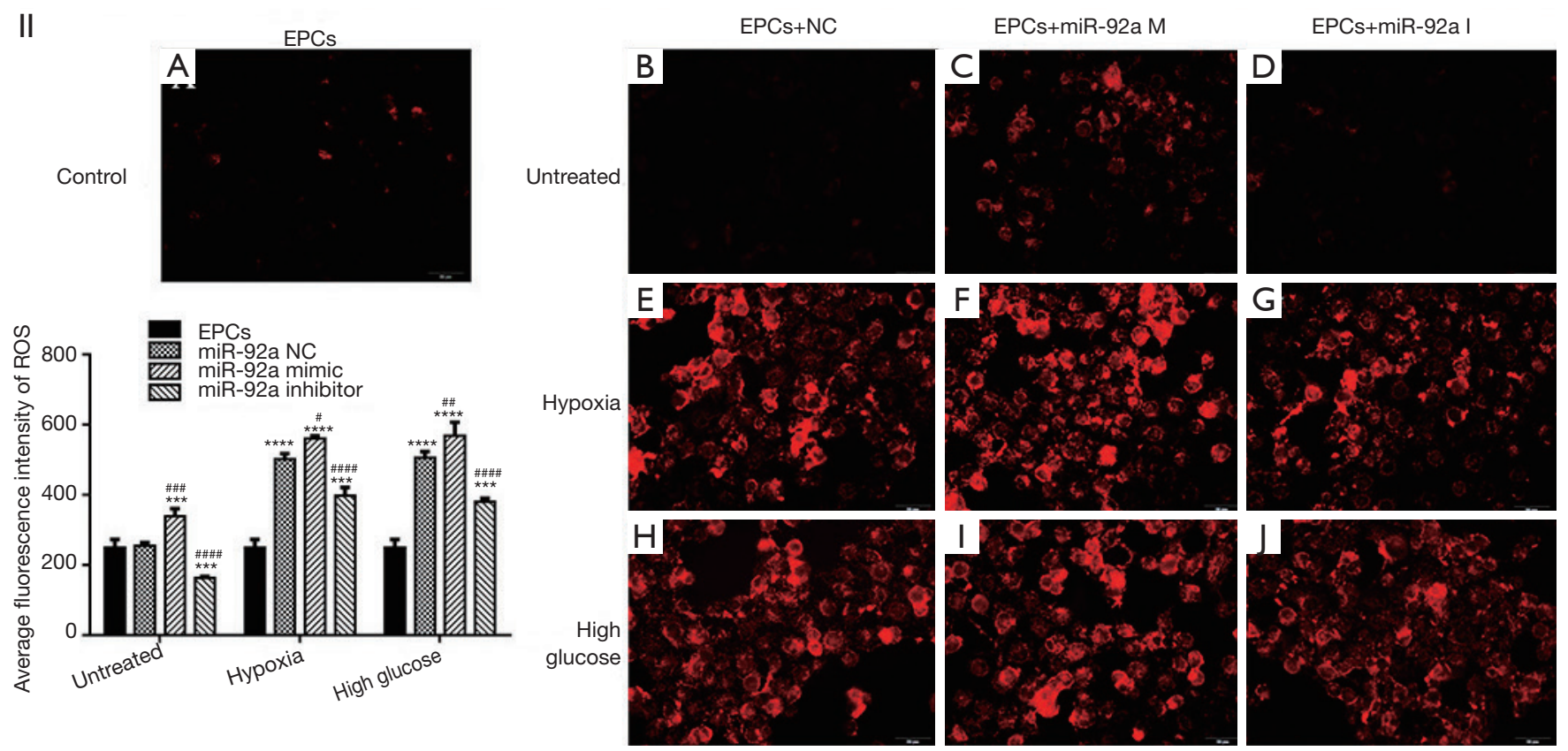

Figure 3 The effect of miR-92a on the expression of adhesive molecules, LDH, NO, ROS of EPCs under the condition of HO or HG. The expression of pecam-1, icam-1, vcam-1 were detected by RT-PCR test (I A-C). LDH and NO in EPCs culture supernatant were examined by ELISA (I D and E). The expression of ROS was detected by ROS Fluorescent Probe-DHE (Dihydroethidium) (II A-J) ${ }^{*}, \mathrm{P}<0.05,{ }^{* * *}, \mathrm{P}<0.01,{ }^{* * *}, \mathrm{P}<0.001,{ }^{* * * *}, \mathrm{P}<0.0001$ vs. control group; ${ }^{\#}, \mathrm{P}<0.05,{ }^{\# \#}, \mathrm{P}<0.01,{ }^{\# \# \#,}, \mathrm{P}<0.001,{ }^{\# \# \#}, \mathrm{P}<0.0001$ vs. miR-92a NC group). 
miR-92a mimic decrease the NO level in all three groups compared with miR-92a NC group (Figure 3I E). The expression of ROS was detected by ROS Fluorescent ProbeDHE (Dihydroethidium). EPCs stimulated by HO or HG produced much more ROS. miR-92a inhibitor inhibited the production of ROS, while miR-92a mimic increase the ROS level in all three groups compared with miR-92a NC group (Figure 3II). These results indicated that $\mathrm{HO}$ and $\mathrm{HG}$ could impair the integrity and promote oxidative stress of EPCs.

The expression of FLK-1 and $\mathrm{vWF}$ were detected by immunofluorescence. The expression of FLK-1 and $\mathrm{vWF}$ were inhibited by treatment of HO or HG. miR-92a mimic further inhibited the expression of FLK-1 and $\mathrm{vWF}$, however, miR-92a inhibitor improved their expression (Figure $4 A, B$ ). These results indicated that $\mathrm{HO}$ and $\mathrm{HG}$ could reduce the expression of FLK-1 and $\mathrm{vWF}$, and knockdown miR-92a expression could restore the expression of FLK-1 and $\mathrm{vWF}$ and promote the differentiation of EPCs.

\section{Regulatory effect of miR-92a on the expression and activity of GDF11}

To investigate whether miR-92a mediates the effects of GDF11, GDF11 expression was detected at the mRNA and protein levels by RT-PCR and Western Blot on EPCs when knockdown or over-expression of miR-92a. mRNA and protein expression increased significantly after miR-92a knockdown and reduced after miR-92a overexpression as compared with control groups. siGDF11 can effectively reverse the expression of GDF11 induced by miR-92a inhibitor, and rGDF11 can effectively rescue the expression of GDF11 induced by miR-92a mimic (Figure $5 A, B$ ). miR-92a can knockdown or over-express by mimic or inhibitor (Figure 5C).

Dual-luciferase reporter assay of EPCs was applied to determine whether miR-92a directly targets GDF11. EPCs were treated with pmirGLO-GDF11-WT, followed by incubation with an miR-92a mimic or inhibitor. The luciferase activity was detected. Over-expression of miR92a inhibited the activity of luciferase, and the activity of luciferase increased when incubated with miR-92a inhibitor in wild type group. When EPCs incubated with miR-92a (mimics or inhibitor) and pmirGLO-GDF11-MUT, the activity of luciferase increased, however, the inhibition effect of miR-92a mimics on the activity of luciferase disappeared when the target site of GDF11 mutated (Figure 5D). These results indicated that miR-92a has direct target effect on GDF11.

\section{MiR-92a regulated the proliferation, apoptosis, migration, and tube formation of EPCs by targeting GDF11}

In MTT test, the proliferation of EPCs had no significant change by inhibiting or over-expressing of miR-92a, however, the proliferation of EPCs was inhibited by GDF11 siRNA (siGDF11) and improved by recombined GDF11 (rGDF11) (Figure 6A). The results consistent with the previous results, but additionally, the results here indicated that GDF11 can promote the proliferation of EPCs. miR92a inhibitor decreased the apoptosis rate of EPCs, and the inhibiting effect could be reversed by siGDF11. Overexpression of miR-92a increased the apoptosis rate of EPCs, however, the apoptosis rate dropped when overexpression of miR-92a and GDF11 together (Figure 6B). The migration ability was improved by inhibition of miR-92a, which could be blocked by silencing of GDF11. Over-expression of miR-92a inhibited the migration ability of EPCs, which was rescued by over-expression of GDF11 (Figure 6C). The ability of tube formation was improved by inhibition of miR-92a as well, but it fell back to normal level when treated by combination with siGDF11. Although over-expression of miR-92a had no significant effect on the ability of tube formation, over-expression of GDF11 significantly improved the tube formation ability (Figure 6D). These results indicated that knockdown miR-92a can suppress apoptosis, improve migration and angiogenesis of EPCs by targeting GDF11.

\section{MiR-92a regulated the expression of $L D H, N O, R O S$ of EPCs by targeting GDF11}

The level of NO in supernate of EPCs culture increased after stimulating of miR-92a inhibitor, however, the promotion effect was blocked by siGDF11. Over-expression of miR-92a inhibited the product of NO, which could be rescued by rGDF11 (Figure $7 A$ ). The total LDH had no significant change in EPCs subjected to miR-92a inhibitor or mimic. The total LDH level decreased in miR-92a inhibitor combined with siGDF11 group compared with miR-92a inhibitor alone group, however, LDH level in both groups had no significant change compared with control group. miR-92a inhibitor reduced the level of LDH in EPCs supernate, and most of LHD was kept in EPCs. siGDF11 reversed the ratio of LDH between extraand intra-cell, and increased the supernate LDH. Over- 
A

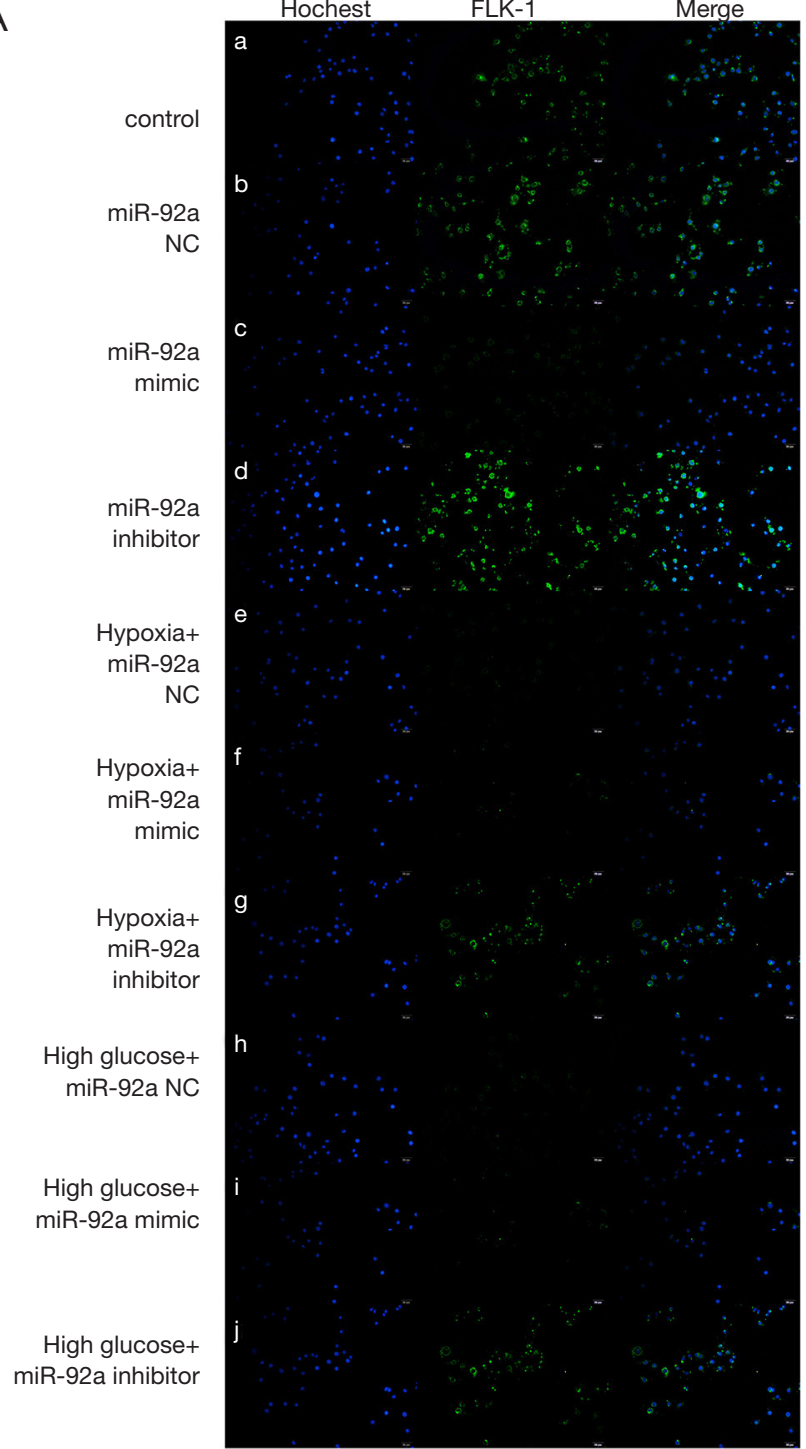

C

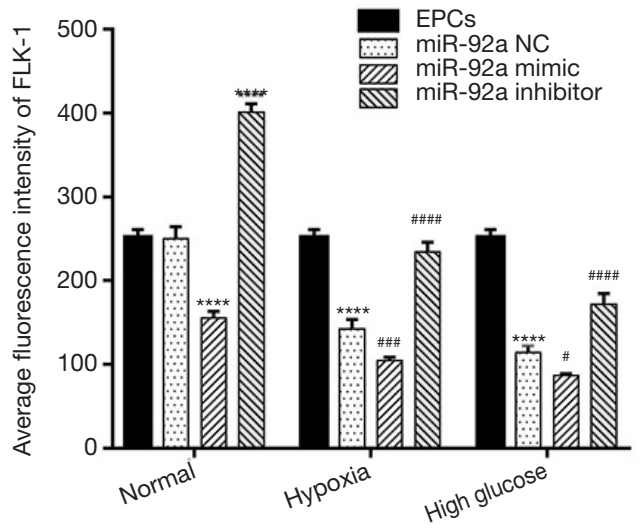

B
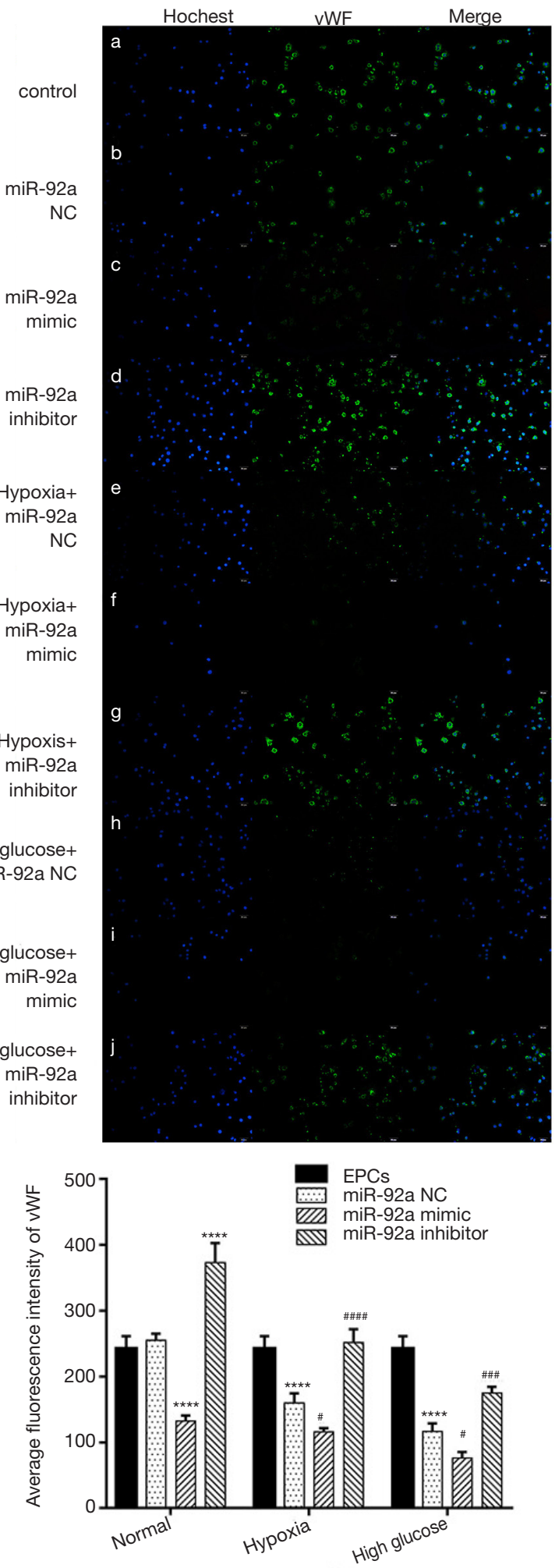

Figure 4 The expression of FLK-1 and vWF were detected by immunofluorescence. (A and C) The expression of FLK-1was inhibited by treatment of $\mathrm{HO}$ or HG, and rescued by miR-92a inhibitor. (B and D) The expression of vWF was inhibited by treatment of $\mathrm{HO}$ or HG, and rescued by miR-92a inhibitor ${ }^{* * * *}, \mathrm{P}<0.0001$ vs. control group; ${ }^{\#}, \mathrm{P}<0.05,{ }^{\# \#,} \mathrm{P}<0.001,{ }^{\# \#+\ldots}, \mathrm{P}<0.0001$ vs. miR-92a NC group). 

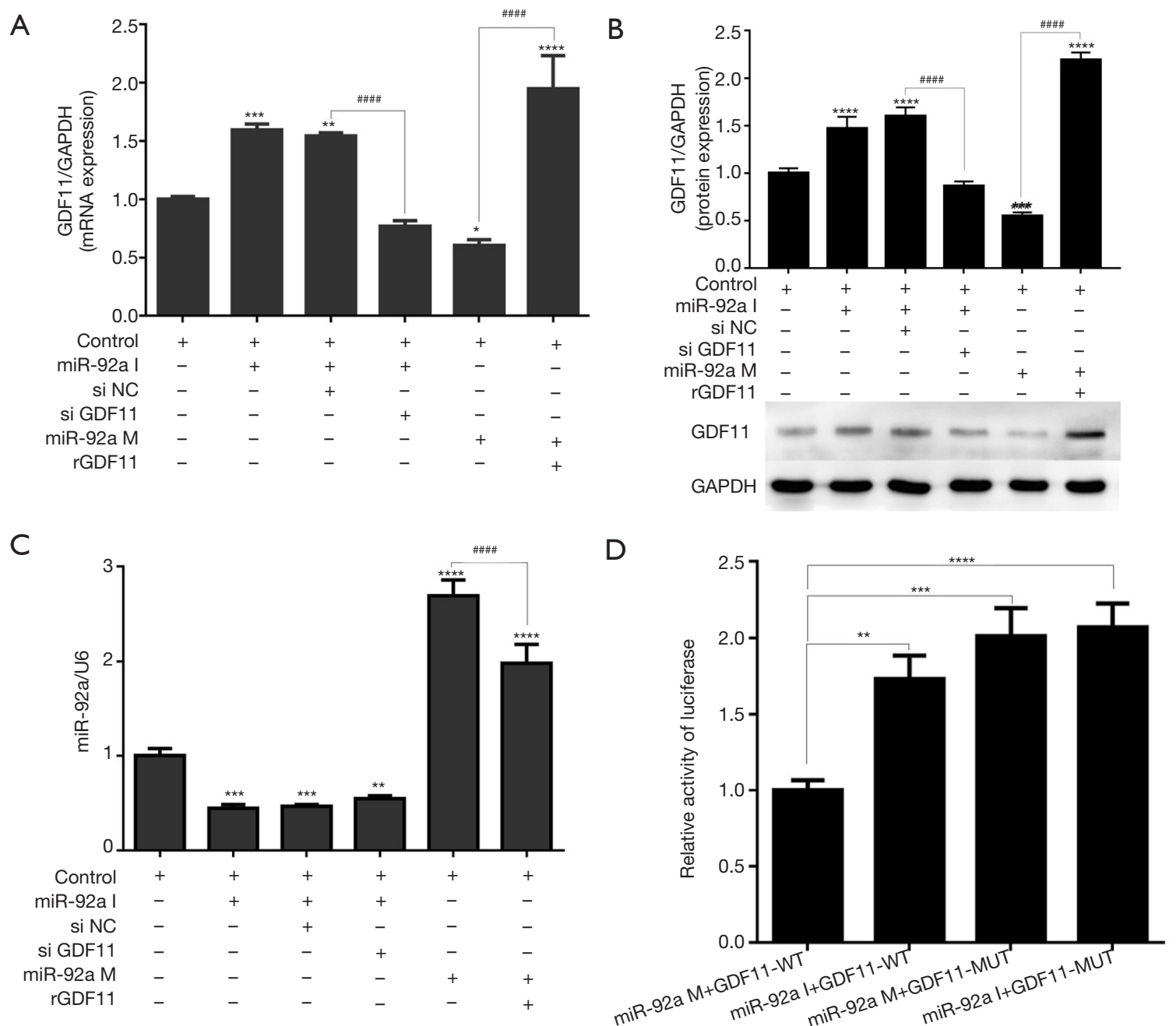

Note: M: mimic; I: inhibitor; WT: wild type; MUT: mutation type

Figure 5 To investigate whether miR-92a mediates the effects of GDF11, GDF11 expression was detected at the mRNA and protein levels. (A and B) mRNA and protein expression increased significantly after miR-92a inhibiting and reduced after miR-92a over-expression as compared with control groups. si GDF11 can effectively inhibitor the expression of GDF11 induced by miR-92a inhibitor, and rGDF11 can effectively increase the expression of GDF11 induced by miR-92a mimic. (C) miR-92a can silence or over-express by mimic or inhibitor. (D) Dual-luciferase reporter assay of EPCs EPCs were treated with blank pGL3 luciferase vector (A) or GDF11-UTR luciferase vector (B), followed by incubation with an miR-92a mimic or inhibitor. The luciferase activity was detected. *, $\mathrm{P}<0.05$ vs. $\mathrm{NC}$ group; ${ }^{*}, \mathrm{P}<0.05$ vs. $\mathrm{NC}$

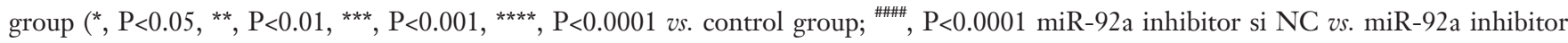
siGDF11 group OR miR-92a mimic $v s$. miR-92a mimic+rGDF11 group). I: inhibitor; M: mimic; si NC: siRNA negative control; si GDF11: si RNA GDF11; rGDF11: recombinant GDF11.

expression of miR-92a promoted the release of $\mathrm{LDH}$ from EPCs, which could be rescued by rGDF11 (Figure $7 B, C, D$ ). Over-expression of miR-92a increased the product of ROS in EPCs, which could be blocked by rGDF11. ROS was inhibited by miR-92a inhibitor, but it was increased when GDF11 was silenced (Figure $7 E$ ). The results showed that knockdown miR-92a can promote the production of
$\mathrm{NO}$ and suppress the release of LDH from EPCs and the expression of ROS by targeting GDF11.

\section{Expression of SMAD2/3, FAK, Akt, and eNOS}

The further mechanism research was performed by investigating the proteins phosphorylation of GDF11 

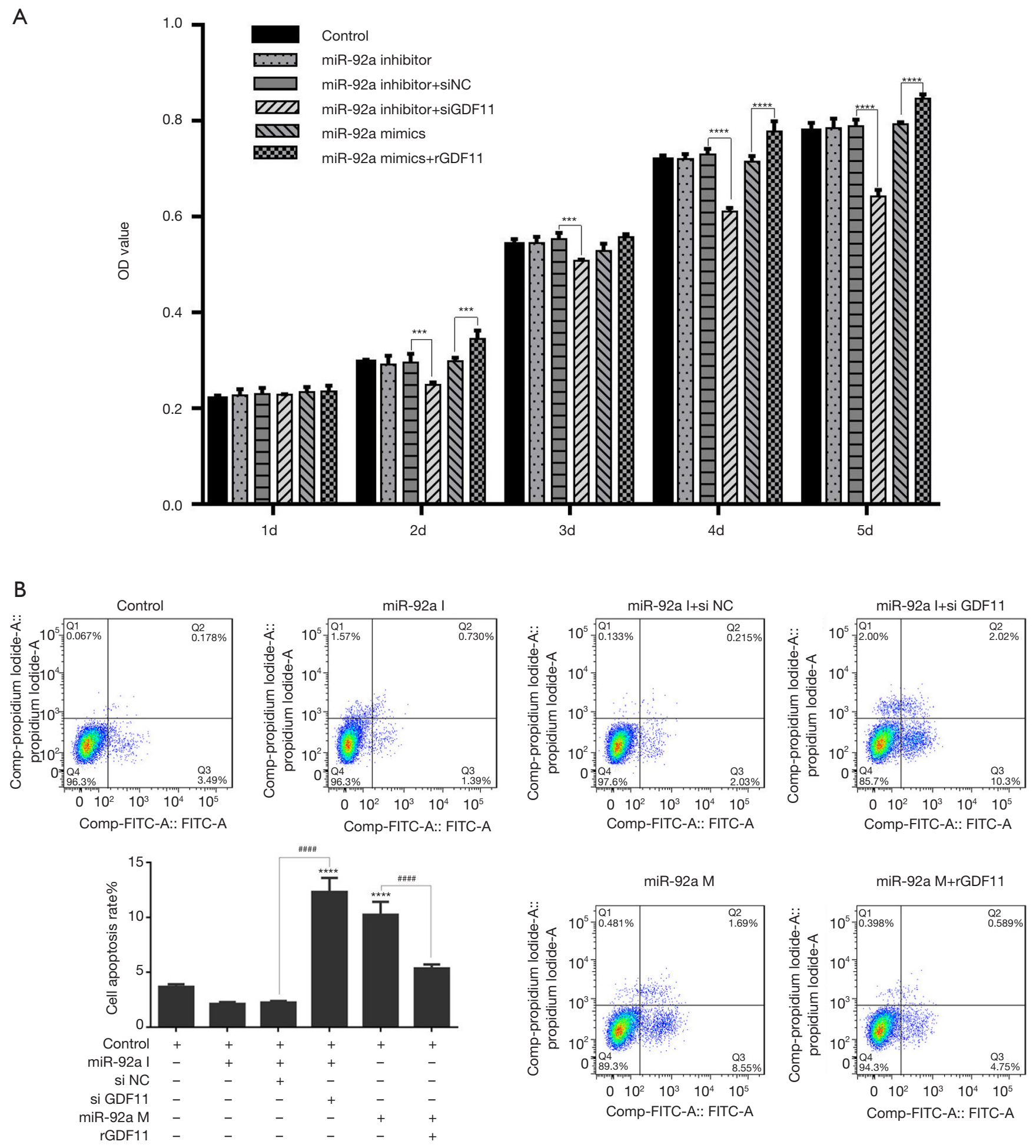

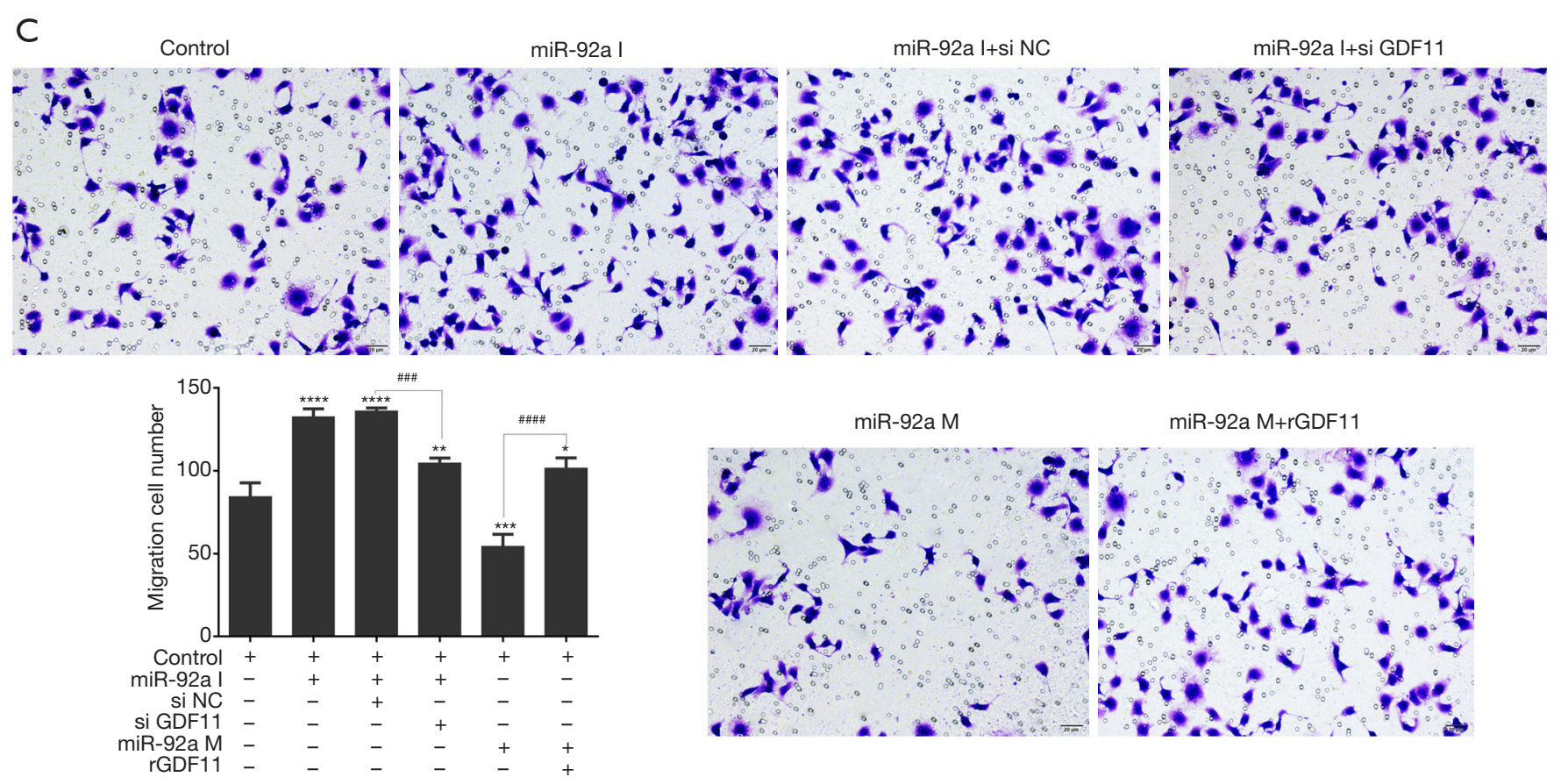

D

Control

miR-92a I

miR-92a I+si NC

miR-92a I+si GDF11
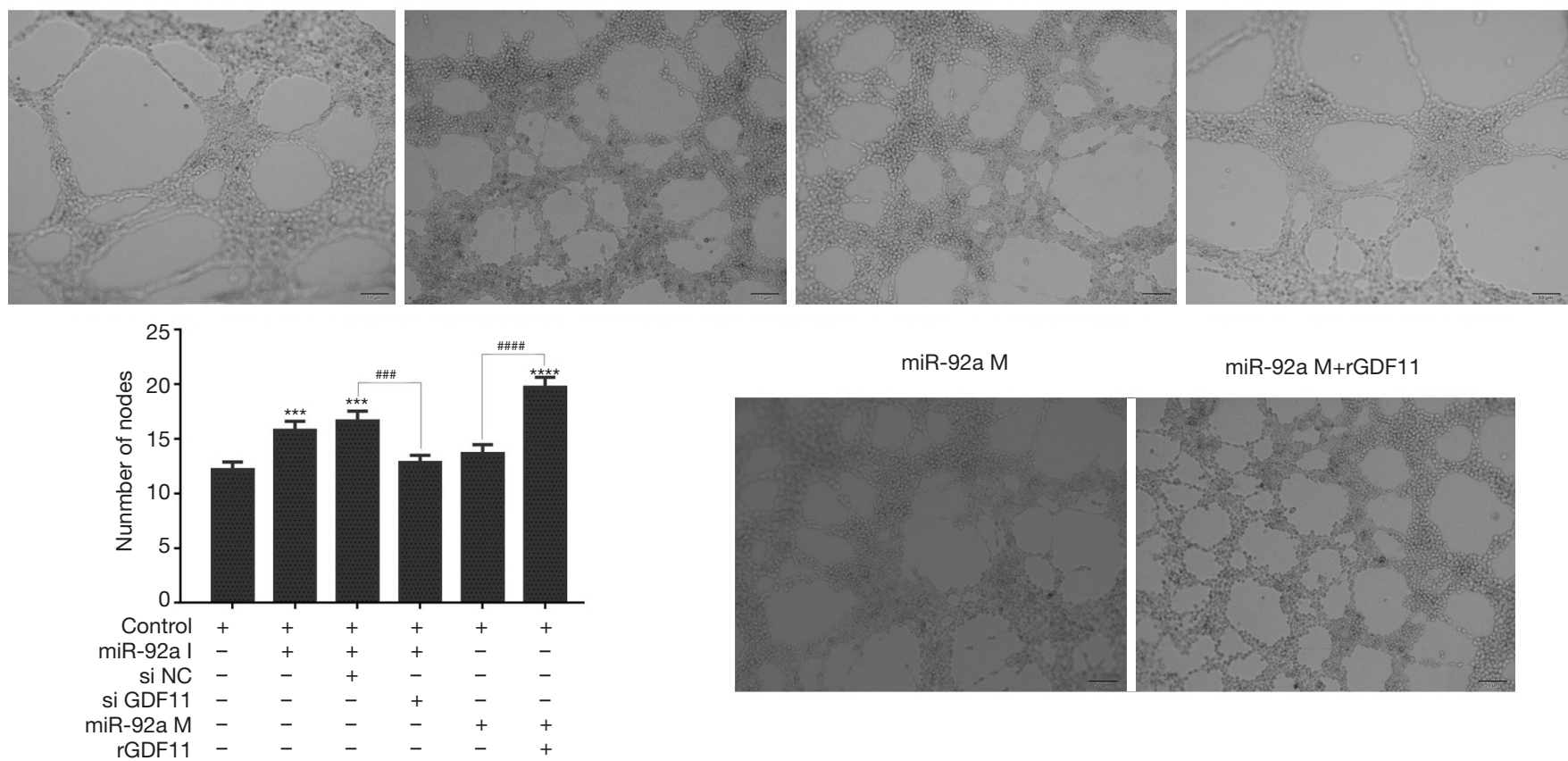

miR-92a M

miR-92a M+rGDF11
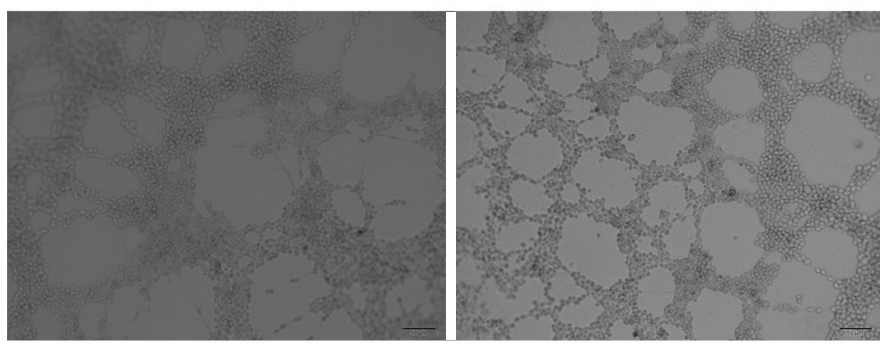

Figure 6 Cell proliferation, apoptosis, migration, tube formation after various treatments EPCs underwent miR-92a inhibition or combined with GDF11 siRNA and miR-92a mimic and recombinant GDF11 (A) MTT assay was applied to detected proliferation of EPCs (***, P<0.001, ****, $\mathrm{P}<0.0001$, si NC vs. si GDF11 in miR-92a inhibitor groups, and mimic vs. mimic with rGDF11). (B) Cells were harvested for the detection of apoptotic cells by flow cytometry (***, $\mathrm{P}<0.0001$ vs. control group, ${ }^{\# \# \#, ~} \mathrm{P}<0.0001$ si NC vs. si GDF11, and mimic vs. mimic with rGDF11). (C) migration ability of EPCs was tested by transwell assay $\left(^{*}, \mathrm{P}<0.05\right.$, ** $, \mathrm{P}<0.01,{ }^{* * *}, \mathrm{P}<0.001$, ****, $\mathrm{P}<0.0001$ vs. control group; ${ }^{\# \# \#, ~} \mathrm{P}<0.001$, \#\#\#\#, $\mathrm{P}<0.0001$ si NC vs. si GDF11, and mimic vs. mimic with rGDF11) (0.1\% crystal violet, 200×). (D) angiogenesis was evaluated by tube formation test $\left({ }^{* * *}, \mathrm{P}<0.001,{ }^{* * * *}, \mathrm{P}<0.0001\right.$ vs. control group; ${ }^{\# \#,} \mathrm{P}<0.001,{ }^{\# \# \#,} \mathrm{P}<0.0001$ si NC vs. si GDF11, and mimic vs. mimic with rGDF11). I: inhibitor; $\mathrm{M}$ : mimic; si NC: siRNA negative control; si GDF11: si RNA GDF11; rGDF11: recombinant GDF11. 
A

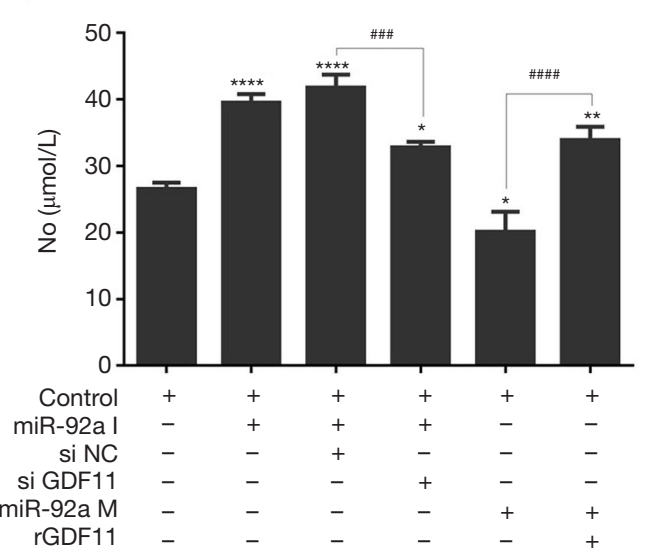

C

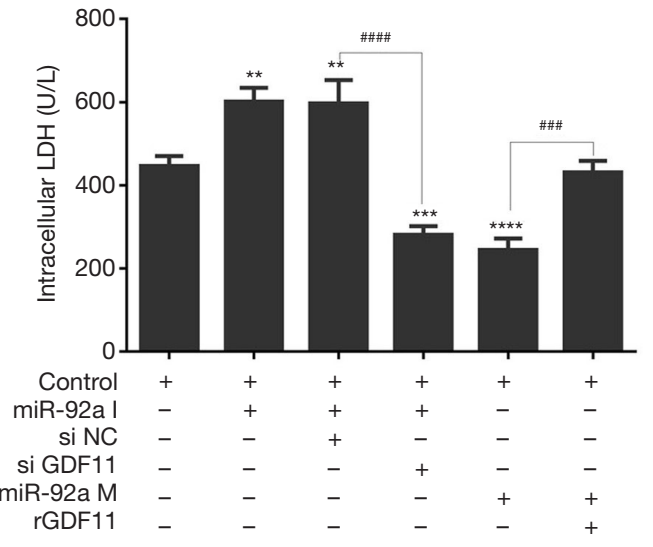

Control
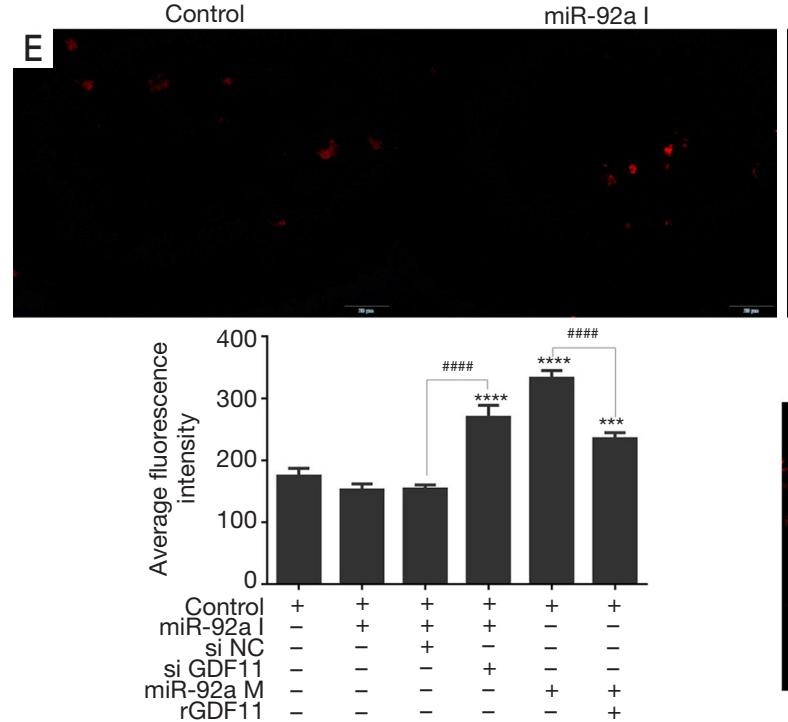

B

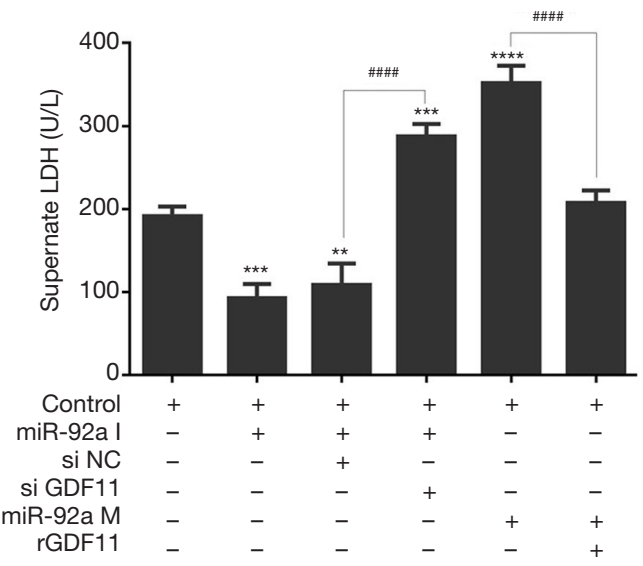

D

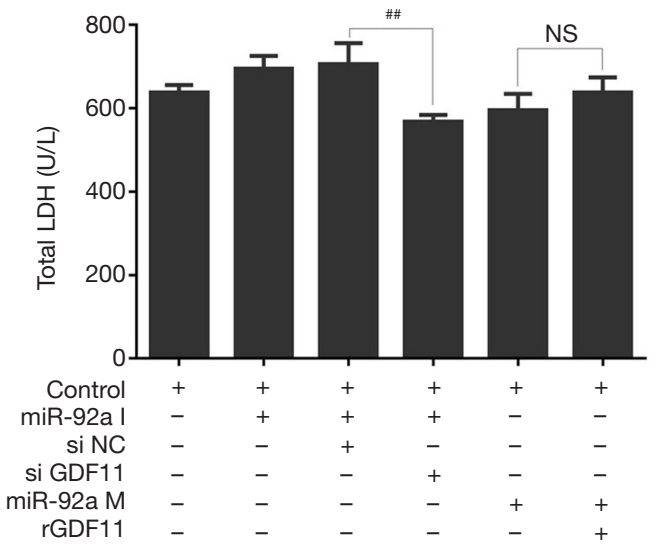

miR-92a I+si NC

miR-92a I+si GDF11

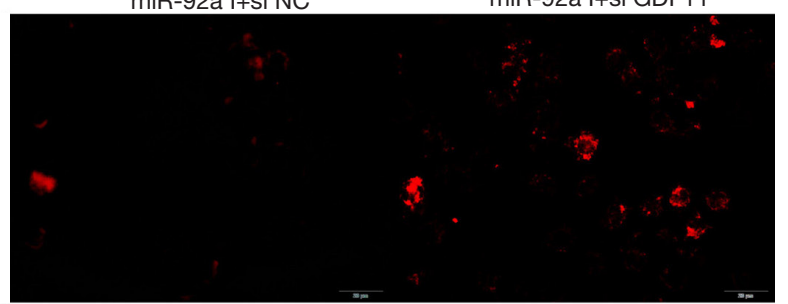

miR-92a M

miR-92a M+rGDF11

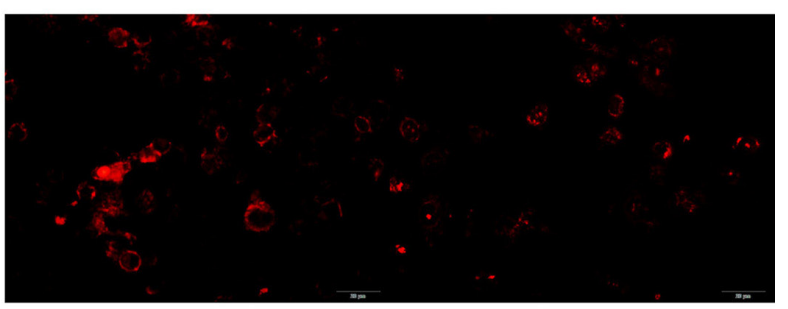

Figure $7 \mathrm{LDH}$ and NO in EPCs after various treatments. EPCs underwent miR-92a inhibition or reversed by siGDF11 and miR-92a mimic or rescued by over-expression of GDF11. Cells were then harvested for the detection of total, intracellular, supernatant LDH by ELISA $\left(^{*}, \mathrm{P}<0.05,{ }^{* *}, \mathrm{P}<0.01,{ }^{* * *}, \mathrm{P}<0.001,{ }^{* * * *}, \mathrm{P}<0.0001\right.$ vs. control group; ${ }^{\# \#, ~}, \mathrm{P}<0.001,{ }^{\# \# \# !}, \mathrm{P}<0.0001$ si NC vs. si GDF11, and mimic $v s$. mimic with rGDF11). Intracellular ROS content in EPCs after various treatments. EPCs underwent the same treatment as before. Cells were then harvested for the detection of intracellular ROS by ROS Fluorescent Probe-DHE (*** $, \mathrm{P}<0.001,{ }^{* * * *}, \mathrm{P}<0.0001$ vs. control group; \#\#\#, $\mathrm{P}<0.0001$ si NC vs. si GDF11, and mimic vs. mimic with rGDF11). I: inhibitor; M: mimic; si NC: siRNA negative control; si GDF11: si RNA GDF11; rGDF11: recombinant GDF11. 

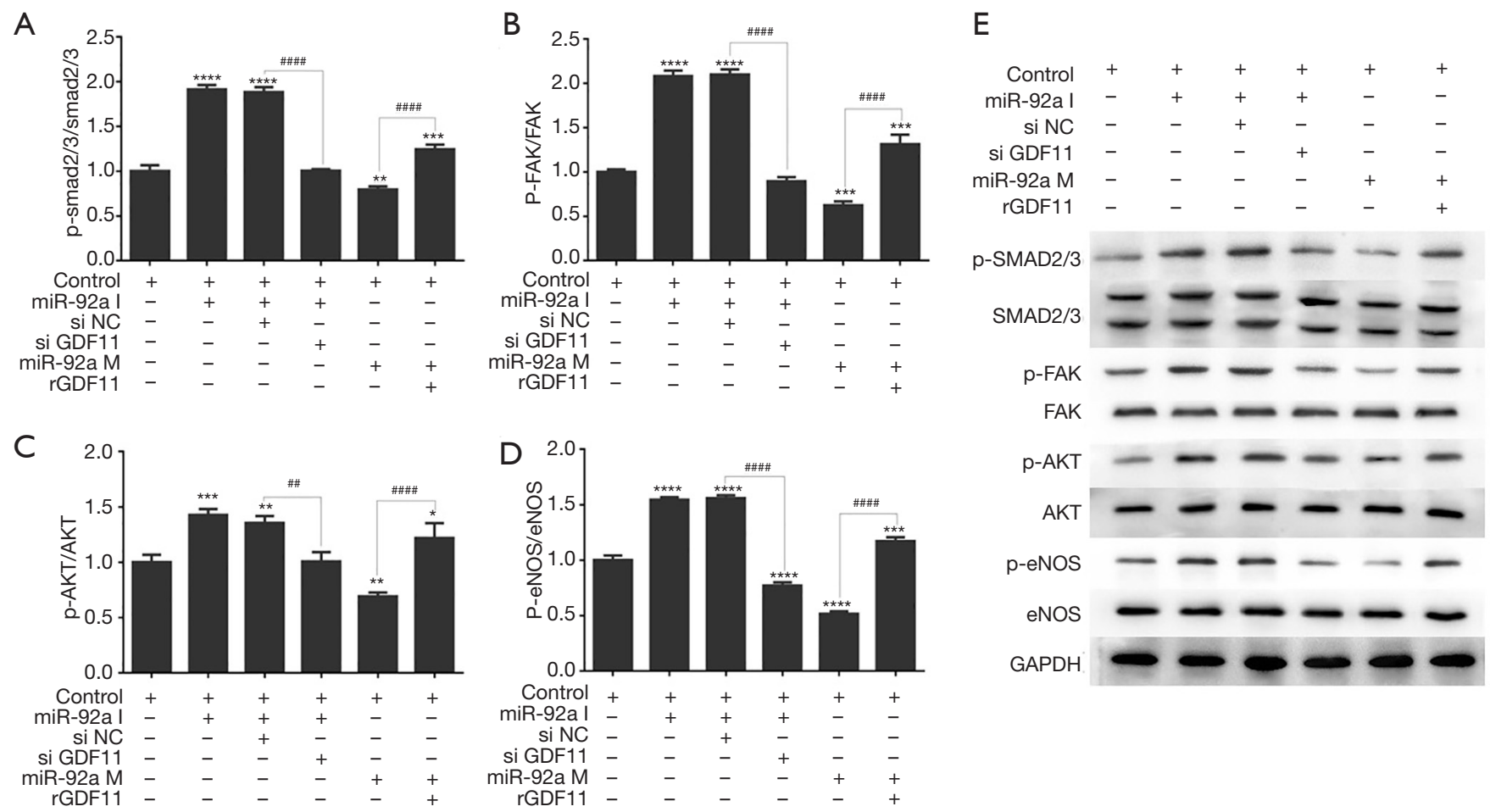

Figure 8 Expression of signal pathway proteins in EPCs underwent miR-92a inhibition or reversed by siGDF11 and miR-92a mimic or rescued by over-expression of GDF11. Protein expression of (A) p-SMAD2/3, SMAD2/3, (B) p-FAK, FAK, (C) p-Akt, Akt, (E) p-eNOS, eNOS was detected after extraction of total protein by western blotting. GAPDH served as an internal reference. The down-expression of p-SMAD2/3, p-FAK, p-Akt, and p-eNOS could be rescued by over-expression of GDF11 when EPCs treated by miR-92a mimic (*, P<0.05, **, $\mathrm{P}<0.01$, ${ }^{* *}, \mathrm{P}<0.001$, ****, $\mathrm{P}<0.0001$ vs. control group; ${ }^{\# \#}, \mathrm{P}<0.01$, \#\#\#, $\mathrm{P}<0.0001$ si NC vs. si GDF11, and mimic vs. mimic with rGDF11). I: inhibitor; M: mimic; si NC: siRNA negative control; si GDF11: si RNA GDF11; rGDF11: recombinant GDF11.

downstream signaling pathway using Western blot. The expression of p-SMAD2/3, p-FAK, p-Akt, and p-eNOS was improved by miR-92a inhibitor compared with control group, which was compromised by siGDF11. Over-expression of miR-92a significantly decreased the expression of $\mathrm{p}-\mathrm{SMAD} 2 / 3$, p-FAK, p-Akt, and p-eNOS, which was rescued by rGDF11 compared between mimic group and mimic+rGDF11group (Figure 8). The results indicated that miR-92a inhibitor could activated SMAD2/3/FAK/Akt/eNOS signal pathway via improving the expression of GDF11.

\section{Discussion}

In this study, we investigated the effects of miR-92a on EPCs under normal and pathological conditions, consequently, the action mechanism of miR-92a on EPCs was further investigated. We found that (I) HO or HG increased apoptosis in EPCs, production of LDH and generation of ROS, but decreased the ability of migration and tube formation and generation of NO; (II) inhibiting of miR-92a decreased HO or HG-induced injury of EPCs, whereas miR-92a over-expression had the opposite effect; and (III) the harmful effects of miR-92a may be related to its inhibition of GDF11 and subsequent inactivation of the SMAD2/3/FAK/Akt/eNOS signaling pathway.

Recently, many studies found that EPCs mobilized into circulation from bone marrow played an important role in endothelial repairing, re-endothelialization, and angiogenesis (4-6). However, the number of circulating EPCs is declined and the function is impaired by several pathological factors, such as acute myocardial infarction (AMI), diabetes, hypertension, hyperlipidemia, and so on (3-6,18).

Diabetes mellitus is a chronic metabolic disease characterized by elevated blood glucose. Georgescu et al. (19) reported that the number of EPCs decreased and the function of EPC was impaired in diabetic patients. Studies found that CXCR7 had a key role in regulating adhesion and survival of EPCs $(20,21)$, and over-expression 
of CXCR7 could improve angiogenic function of EPCs in diabetic patients via Akt/GSK-3 $\beta /$ Fyn-Mediated Nrf2 Activation (22).

Although the mobilization of EPCs from BM increased by ischemia disease (ID), the number and function of EPCs was insufficient to the repair of vessel and myocardium (4). Acute ischemia lead to hypoxia microenvironment, and ID patients always complicated with DM, hypertension, and hyperlipidemia, which attributed to the abnormalities of EPCs. In this study, the biological characteristics of EPCs was evaluated under the condition of $\mathrm{HO}$ and HG.

Increasing the number and ameliorating the function of EPCs has become an attractive therapeutic treatment for ID. Many methods were detected to improve the number and function of EPCs, including chemical medicine such as Aliskiren (3), traditional Chinese medicine such as Velvet Antler (4), bioactive protein or factors such as 17ß-estradiol (6), ANGPTL3 (5), GDF11 (10,11), CXCR7 (20-22), microRNAs such as miR-150 (23), miR-126-3p (24) and so on.

Studies have found that multiple microRNAs affect EPCs through different target genes (25). Over-expression of miR-150 improved angiogenesis and proliferation capabilities of EPCs by targeting SRCIN1 both in vitro and in vivo (23). Over-expression of miR-126-3p significantly promoted migration and tube formation of EPCs from ischemic cardiomyopathy patients. In nude rat with ischemic cardiomyopathy induced by ligation of left anterior descending, left ventricular function was improved with smaller infarction size, thicker anterior wall, and less inflammation reaction after transplanted with miR-126-3p overexpressing EPCs (24).

The role of miR-92a in endothelial cells is comparatively clearer. miR-92a expresses in vascular endothelial cells of various tissues. Over-expression of miR-92 a could inhibit the migration and angiogenesis of human umbilical vein endothelial cells (HUVECs) without affecting the proliferation (13). Inhibiting the expression of miR-92a can significantly ameliorate the re-endothelialization repair of damaged vascular endothelium and inhibit the formation of neointima (15). Results in this study also indicated that miR-92a expression increased in EPCs when treated by hypoxia or high glucose. This result was consistent to the previous research outcome that miR92a express in EPCs, and increased in EPCs from CAD patients (17). However, there was no precedent reports about the action effect of miR-92a on biology of EPCs. miR-92a corrects CD34+ cell dysfunction in diabetes in progenitor differentiation (26). In this study, we also investigated the effect of miR-92a on EPCs under the circumstance of physiological and pathological conditions. Inhibiting of miR-92a decreased HO or HG-induced injury of EPCs including inhibiting apoptosis, improving migration and angiogenesis, which was consistent with those observed on HUVECs.

Vascular endothelial growth factor (VEGF) receptor type 2 (FLK-1, VEGFR2) and von Willebrand Factor (vWF) both expressed on the late EPCs (27). Increased levels of FLK-1 positive EPCs have been associated with improved reendothelialization (28). With the EPCs passaged, EPCs gradually differentiated into ECs who mainly expressed the EC-specific markers CD31 and vWF (27). in this study, FLK-1 and vWF in late EPCs were detected. Both $\mathrm{HO}$ and HG reduce the expression of FLK-1 and vWF. Inhibiting of miR-92a could rescue the expression of FLK-1 and $\mathrm{vWF}$ and promote the differentiation of EPCs.

Prediction of database and the results of this study demonstrate that GDF11 is one of the target genes of miR92a. knockdown of miR-92a improves the transcription and translation of GDF11 at the level of mRNA and protein expression. GDF11 has promoting function on EPCs. Consequently knockdown of miR-92a improves the function of EPCs via up-regulating GDF11.

eNOS is the constitutive NOS form in ECs, which regulate the production of $\mathrm{NO}(29)$. NO is a critical endothelial function regulator (30), which regulates both vasorelaxation and angiogenesis under the condition of ischemia. Several studies demonstrate that eNOS and its product NO play an important role in maintaining the protective function of ECs (31) and EPCs (32-34). $\mathrm{PI} 3 \mathrm{~K} / \mathrm{Akt}$ is a canonial signal pathway, which can protect endothelial function by activating its pivotal target eNOS (31). Hyperinsulinemia impaired the tube formation ability of EPCs by inactive of PI3K/Akt/eNOS pathway (34). ATP-binding cassette transporter G1 (ABCG1) could improve the migration, tube formation, differentiation, and re-endothelialization ability of EPCs via Lyn/Akt/eNOS after vascular injury in diabetes model (33). H2S promotes the mobilization of EPCs and re-endothelialization in eNOS-dependent manner following vascular injury (32).

In this study, the ROS, LDH was increased and the NO was reduced in EPCs when treated by HO and HG, but the abnormal expression was reversed by miR-92a knockdown. In order to understanding the underlie mechanism, interaction between miR-92a and GDF11 was investigated 
by dual-luciferase reporter assay and further verified by RT-PCR and Western blot. Results showed that miR92a targeted inhibition of GDF11. The activity of eNOS and Upstream regulatory pathway (SMAD, FAK, PI3K/ AKT) were also tested. In EPCs, knockdown of miR-92a could increase the expression of GDF11 and promote the phosphorylation of SMAD2/3, FAK, AKT, eNOS, which indicated that miR-92a regulated the function of EPCs by targeting GDF11 and downstream signaling pathway SMAD2/3/FAK/Akt/eNOS.

Several possible limitations of this study should be considered. After miR-92a knockdown or over-expression, we detected biology of EPCs following HO and HG, but whether GDF11 knockdown or over-expression affects the biology of EPCs was not presented (data not shown). In mechanism study, interaction between miR-92a and GDF11 was investigated in untreated EPCs. Whether miR-92a regulated the function of EPCs under the condition of $\mathrm{HO}$ and HG by targeting GDF11 was unknown. SMAD2/3 was the canonial signal pathway of GDF11 and the PI3K/AKT was the noncanonial signal pathway (7), in addition FAK could be activated by SMAD2/3 (35), and FAK and PI3K/ AKT could activate each other (36). Regulatory relationship among SMAD2/3, FAK, PI3K/AKT was not investigated in detail. Thus, our results need to be confirmed by additional experiments and in animal models in the future.

Taken together, our findings suggest that inhibiting miR92a may up-regulate GDF11 to activate the SMAD2/3/ FAK/Akt/eNOS signaling pathway, exerting protective effects on $\mathrm{HO}$ or HG-induced injury to EPCs.

\section{Acknowledgments}

Funding: This study was supported by Natural Science Foundation of China (81670463) and Shanghai Science and Technology Committee Foundation (16441907800).

\section{Footnote}

Conflicts of Interest: The authors have no conflicts of interest to declare.

Ethical Statement: The whole study was approved by the Ethics Committee of Nantong First People's Hospital. The authors are accountable for all aspects of the work in ensuring that questions related to the accuracy or integrity of any part of the work are appropriately investigated and resolved.

\section{References}

1. Wang ZC, Qi J, Liu LM, et al. Valsartan reduces AT1AA-induced apoptosis through suppression oxidative stress mediated ER stress in endothelial progenitor cells. Eur Rev Med Pharmacol Sci 2017;21:1159-68.

2. Asahara T, Masuda H, Takahashi T, et al. Bone marrow origin of endothelial progenitor cells responsible for postnatal vasculogenesis in physiological and pathological neovascularization. Circ Res 1999;85:221-8.

3. Chang TT, Wu TC, Huang PH, et al. Aliskiren directly improves endothelial progenitor cell function from Type II diabetic patients. Eur J Clin Invest 2016;46:544-54.

4. Li Y, Wang Z, Mao M, et al. Velvet Antler Mobilizes Endothelial Progenitor Cells to Promote Angiogenesis and Repair Vascular Endothelial Injury in Rats Following Myocardial Infarction. Front Physiol 2019;9:1940.

5. Luo F, Wu P, Chen J, et al. ANGPTL3 possibly promotes cardiac angiogenesis through improving proangiogenic ability of endothelial progenitor cells after myocardial infarction. Lipids Health Dis 2018;17:184.

6. Yuan Z, Kang L, Wang Z, et al. 17beta-estradiol promotes recovery after myocardial infarction by enhancing homing and angiogenic capacity of bone marrow-derived endothelial progenitor cells through ERalpha-SDF-1/ CXCR4 crosstalking. Acta Biochim Biophys Sin (Shanghai) 2018;50:1247-56.

7. Jamaiyar A, Wan W, Janota DM, et al. The versatility and paradox of GDF 11. Pharmacol Ther 2017;175:28-34.

8. Heidecker B, Olson K, Beatty A, et al. Low levels of growth differentiation factor 11 and high levels of its inhibitor follistatin-like 3 are associated with adverse cardiovascular outcomes in humans. J Am Coll Cardiol 2015;65:A999.

9. Olson KA, Beatty AL, Heidecker B, et al. Association of growth differentiation factor 11/8, putative anti-ageing factor, with cardiovascular outcomes and overall mortality in humans: analysis of the Heart and Soul and HUNT3 cohorts. Eur Heart J 2015;36:3426-34.

10. Finkenzeller G, Stark GB, Strassburg S. Growth differentiation factor 11 supports migration and sprouting of endothelial progenitor cells. J Surg Res 2015;198:50-6.

11. Zhang J, Li Y, Li H, et al. GDF11 Improves Angiogenic Function of EPCs in Diabetic Limb Ischemia. Diabetes 2018;67:2084-95.

12. Ou Z, Wang Y, Chen J, et al. Estrogen receptor beta promotes bladder cancer growth and invasion via alteration of miR-92a/DAB2IP signals. Exp Mol Med 2018;50:152. 
13. Bonauer A, Carmona G, Iwasaki M, et al. MicroRNA-92a controls angiogenesis and functional recovery of ischemic tissues in mice. Science 2009;324:1710-3.

14. Casadei L, Calore F, Creighton CJ, et al. ExosomeDerived miR-25-3p and miR-92a-3p Stimulate Liposarcoma Progression. Cancer Res 2017;77:3846-56.

15. Daniel JM, Penzkofer D, Teske R, et al. Inhibition of miR92a improves re-endothelialization and prevents neointima formation following vascular injury. Cardiovasc Res 2014;103:564-72.

16. Gou L, Zhao L, Song W, et al. Inhibition of miR-92a Suppresses Oxidative Stress and Improves Endothelial Function by Upregulating Heme Oxygenase- 1 in db/db Mice. Antioxid Redox Signal 2018;28:358-70.

17. Zhang Q, Kandic I, Kutryk MJ. Dysregulation of angiogenesis-related microRNAs in endothelial progenitor cells from patients with coronary artery disease. Biochem Biophys Res Commun 2011;405:42-6.

18. Lan H, Wang Y, Yin T, et al. Progress and prospects of endothelial progenitor cell therapy in coronary stent implantation. J Biomed Mater Res B Appl Biomater 2016;104:1237-47.

19. Georgescu A, Alexandru N, Constantinescu A, et al. The promise of EPC-based therapies on vascular dysfunction in diabetes. Eur J Pharmacol 2011;669:1-6.

20. Dai X, Tan Y, Cai S, et al. The role of CXCR7 on the adhesion, proliferation and angiogenesis of endothelial progenitor cells. J Cell Mol Med 2011;15:1299-309.

21. Yan X, Cai S, Xiong X, et al. Chemokine receptor CXCR7 mediates human endothelial progenitor cells survival, angiogenesis, but not proliferation. J Cell Biochem 2012;113:1437-46.

22. Dai X, Yan X, Zeng J, et al. Elevating CXCR7 Improves Angiogenic Function of EPCs via Akt/GSK-3beta/FynMediated Nrf2 Activation in Diabetic Limb Ischemia. Circ Res 2017;120:e7-23.

23. Wang $W$, Zhu X, Du X, et al. MiR-150 promotes angiogensis and proliferation of endothelial progenitor cells in deep venous thrombosis by targeting SRCIN1. Microvasc Res 2019;123:35-41.

24. Li H, Liu Q, Wang N, et al. Transplantation of Endothelial Progenitor Cells Overexpressing miR-126-3p Improves Heart Function in Ischemic Cardiomyopathy. Circ J 2018;82:2332-41.

25. Scott E, Loya K, Mountford J, et al. MicroRNA regulation of endothelial homeostasis and commitment-implications for vascular regeneration strategies using stem cell therapies. Free Radic Biol Med 2013;64:52-60.
26. Bhatwadekar AD, Yan Y, Stepps V, et al. miR-92a Corrects CD34+ Cell Dysfunction in Diabetes by Modulating Core Circadian Genes Involved in Progenitor Differentiation. Diabetes 2015;64:4226-37.

27. Shoeibi S, Mohammadi S, Sadeghnia HR, et al. Determine exogenous human DDAH2 gene function in rabbit bone marrow-derived endothelial progenitor cells in vitro. Cell Biochem Funct 2017;35:69-76.

28. Steinmetz M, Brouwers C, Nickenig G, et al. Synergistic effects of telmisartan and simvastatin on endothelial progenitor cells. J Cell Mol Med 2010;14:1645-56.

29. Tejero J, Shiva S, Gladwin MT. Sources of Vascular Nitric Oxide and Reactive Oxygen Species and Their Regulation. Physiol Rev 2019;99:311-79.

30. Mahmoud AM, Ali MM, Miranda ER, et al. Nox2 contributes to hyperinsulinemia-induced redox imbalance and impaired vascular function. Redox Biol 2017;13:288-300.

31. Saad MI, Abdelkhalek TM, Saleh MM, et al. Insights into the molecular mechanisms of diabetes-induced endothelial dysfunction: focus on oxidative stress and endothelial progenitor cells. Endocrine 2015;50:537-67.

32. Hu Q, Ke X, Zhang T, et al. Hydrogen sulfide improves vascular repair by promoting endothelial nitric oxide synthase-dependent mobilization of endothelial progenitor cells. J Hypertens 2019;37:972-84.

33. Shi Y, Lv X, Liu Y, et al. Elevating ATP-binding cassette transporter G1 improves re-endothelialization function of endothelial progenitor cells via Lyn/Akt/eNOS in diabetic mice. FASEB J 2018;32:6525-36.

34. Tan Q, Li Y, Li X, et al. Hyperinsulinemia impairs functions of circulating endothelial progenitor cells. Acta Diabetol 2019;56:785-95.

35. Ding Q, Subramanian I, Luckhardt TR, et al. Focal adhesion kinase signaling determines the fate of lung epithelial cells in response to TGF-beta. Am J Physiol Lung Cell Mol Physiol 2017;312:L926-35.

36. Li M, Sun X, Ma L, et al. SDF-1/CXCR4 axis induces human dental pulp stem cell migration through FAK/ PI3K/Akt and GSK3beta/beta-catenin pathways. Sci Rep 2017;7:40161.

Cite this article as: Huang HT, Liu ZC, Wu KQ, Gu SR, Lu TC, Zhong CJ, Zhou YX. MiR-92a regulates endothelial progenitor cells (EPCs) by targeting GDF11 via activate SMAD2/3/FAK/Akt/eNOS pathway. Ann Transl Med 2019;7(20):563. doi: 10.21037/atm.2019.09.35 